\title{
Wellposedness in the Lipschitz class for a quasi-linear hyperbolic system arising from a model of the atmosphere including water phase transitions
}

\author{
Davide Ascoli and Steave C. Selvaduray
}

\begin{abstract}
In this paper wellposedness is proved for a diagonal quasilinear hyperbolic system containing integral quadratic and Lipschitz continuous terms which prevent from looking for classical solutions in Sobolev spaces. It is the hyperbolic part of the system introduced in [Selvaduray and Fujita Yashima on Atti dell'Accademia delle Scienze di Torino 2011] as a model for air motion in $\mathbf{R}^{3}$ including water phase transitions. Unknown functions are: the densities $\rho$ of dry air, $\pi$ of water vapor, $\sigma$ and $\nu$ of water in the liquid and solid state, dependent also on the mass $m$ of the droplets or ice particles. Air velocity $v$ and temperature $T$ are assumed to be known. Solutions $(\rho, \pi, \sigma, \nu)$ lie in $L^{\infty}(] 0, \tau^{*}\left[; W^{1, \infty}(\Omega)\right)^{2} \times$ $L^{\infty}(] 0, \tau^{*}\left[; W^{1, \infty}\left(\Omega^{+}\right)\right)^{2}$, where $\left.\Omega^{+}=\Omega \times\right] 0,+\infty\left[, \Omega \subset \mathbf{R}^{3}\right.$ is open and bounded, and $\tau^{*}$ is sufficiently small; they depend continuously on initial data, temperature and velocities, which are tangent to $\partial \Omega$; they lie also in $W^{1, q}(] 0, \tau^{*}\left[; L^{\infty}(\Omega)\right)^{2} \times W^{1, q}(] 0, \tau^{*}\left[; L^{\infty}\left(\Omega^{+}\right)\right)^{2}$, where $q \in[1, \infty]$.
\end{abstract}

Mathematics Subject Classification (2000). 35Q35, 35L60, 76T30, 76N10, 35L45, 76T10.

Keywords. Model of the atmosphere, Phase transitions, Quasi-linear hyperbolic system, Integral semilinear terms, Solutions in the Lipschitz class.

\section{Introduction}

It would be of crucial importance, for mathematical modelling of atmospheric and meteorological phenomena, to have a description including water phase transitions - hence, possibly, water droplets and ice particles - of air motion. Nonetheless, the setting of the mathematical study of this problem has been scarcely developed until now. A mathematical model for air motion including phase transition between water liquid and gaseous phases was proposed in 
[13]. However, a direct study of that model gave rise to an excessively hard mathematical challenge.

Selvaduray and Fujita Yashima introduced then in [19] a model consisting of a system of equations for air motion in three-dimensional space, including water phase transitions from one to any other of its three states: gaseous, liquid and solid; this model was the complete version of the simplified one discussed in [4], in which solid state was excluded, and space was one-dimensional.

The model described in [19] represents a sufficiently detailed mathematical description of the physical phenomena involved (yet, interaction with heat radiation would require further investigations, see [18] and [12] for a result on the stationary case). Nonetheless, in [19], as well as in [4], a (local in time) existence and uniqueness theorem was proved for a modified problem in which vapor density is replaced by a local average of it.

In this paper, instead, we intend to make a first step in the direct studywithout smoothing the vapor density - of the model discussed in [19]: namely, we suppose that air velocity $v$ and temperature $T$ are known at every time $t$ and point $x$ (whereas in [19] they satisfy parabolic equations), and prove wellposedness for the hyperbolic part of the system introduced in [19]. This subsystem is diagonal quasilinear hyperbolic, and it contains, in its semilinear part, both quadratic collisional integrals and Lipschitz continuous terms; these latter prevent looking for classical solutions in higher order Sobolev spaces of square-integrable functions (and indeed we show, in Sect. 7, that solutions may be not differentiable in the space variables); otherwise one could apply Kato's results of [15], which concern also systems with terms in which dependence on the solution is not pointwise. Diagonal (or diagonalizable) quasilinear hyperbolic systems with Lipschitz continuous bounded coefficients and inhomogeneous terms (not including integral terms) were studied in the whole space by Cinquini Cibrario in [8] (see also [10] for a generalization to a case with discontinuous coefficients), where wellposedness in the Lipschitz class was proved; see also [14] for a semigroup approach in the Lipschitz class, [9] and [7] for more classical results, [16] and [17] for a semigroup approach to solutions of class $C^{1}$.

The unknown functions of our system are:

$\rho$, the density of dry air (that can be dealt separately),

$\pi$, the density of water vapor;

$\sigma$, the density of water in the liquid state, also dependent on the mass $m$ of the droplets: $\sigma(t, x, m)$ represents, at time $t$, the total mass, per unit volume centered at $x$, of the water contained in droplets of mass $m$; and, analogously, $\nu$, the density of water at the solid state, also dependent on the mass $m$ of ice particles.

A description of the model is given in Sect. 2.

For the aforesaid system we prove local in time wellposedness for a Cauchy problem whose initial data are Lipschitz continuous functions of $x$ and $m$, where $x \in \Omega-$ a bounded open set of $\mathbf{R}^{3}$ with strongly Lipschitz continuous boundary $\partial \Omega$ - and $m \in] 0,+\infty[$; velocities are supposed to be tangent to $\partial \Omega$. The solutions $(\rho, \pi, \sigma, \nu)$ of our system lie in $L^{\infty}(] 0, \tau^{*}\left[; W^{1, \infty}(\Omega)\right)^{2} \times$ 
$L^{\infty}(] 0, \tau^{*}\left[; W^{1, \infty}\left(\Omega^{+}\right)\right)^{2}$, where $\left.\Omega^{+}=\Omega \times\right] 0,+\infty\left[\right.$, and $\tau^{*}$ is sufficiently small, but $\sigma$ and $\nu$ have bounded support; in this space we also prove the continuous dependence of solutions on initial data, velocities and temperature; our solutions have some regularity in time too, since they lie also in $W^{1, q}(] 0, \tau^{*}\left[; L^{\infty}(\Omega)\right)^{2} \times W^{1, q}(] 0, \tau^{*}\left[; L^{\infty}\left(\Omega^{+}\right)\right)^{2}$, where $q \in[1, \infty]$ depends on a time regularity of velocities and temperature. In our main result the velocities $v$ of air, $u$ of droplets and $w$ of ice particles are supposed to be independent of each other, but in Sect. 7 we briefly discuss which relationships may be assumed to exist among them and how our main theorem can be applied. Our chief result is stated in Sect. 3 .

In order to prove our theorem, we first consider a linearization of the system, which is a linear hyperbolic diagonal one that we study in Sect. 5 and whose coefficients are Lipschitz continuous in $x$ and $m$. This linearization is similar to the one adopted in [19], and has the advantage that the inhomogeneous terms are non-negative; this allows us to prove that the densities $\rho$, $\pi, \sigma$ and $\nu$ are non-negative. It is well known (see e.g. [8]) that integration along characteristics gives solutions of linear Cauchy problems; nonetheless, our problem is considered on a bounded open set, and we also need estimates; we are thus led to prove more specific preliminary results in Sect. 4; the main proof we perform is based on $L^{\infty}$-estimates as limits of $L^{p}$-estimates (Tadmor used this procedure - which could be useful also in dealing with non-diagonal systems - in a simple situation in [20]), regularization of the coefficients and of the inhomogeneous terms, and converging subsequences. This way, we avoid a direct use of integration along characteristics. The use of $L^{p}$-estimates could even prepare us for further results.

In order to find the solution of the quasi-linear problem for $(\pi, \sigma, \nu)$ - the equation in $\rho$ being linear - we then apply the contraction theorem in a closed subset of $L^{\infty}\left(\left[0, \tau^{*}\right] ; W^{1, \infty}(\Omega)\right) \times L^{\infty}\left(\left[0, \tau^{*}\right] ; W^{1, \infty}\left(\Omega^{+}\right)\right)^{2}$. The contraction is with respect to the $L^{\infty}$-norm.

We need, to this end, suitable majorizations of the $L^{\infty}$-norms of the solutions of the linearized problem and of their derivatives with respect to $x$ and to $m$.

Last, we prove the locally Lipschitz continuous dependence of the solution of the quasilinear problem on initial data, temperature and velocities. The proof of our main result is given in Sect. 6.

Notations have been chosen in such a way as to avoid any confusion in comparing with [19].

The authors are very grateful to Professor Hisao Fujita Yashima for having introduced them into this topic, for his encouragement, and for the interest he has demonstrated for this research.

\section{Description of the model}

The quasilinear system for which we are going to study the Cauchy problem is given by equations (3.1)-(3.4) of [19] — where a detailed explanation of the model is given - in the unknown scalar functions $\rho(t, x), \pi(t, x), \sigma(t, x, m)$ and 
$\nu(t, x, m)$, and can be written in the form

$$
(Q)\left\{\begin{array}{l}
\frac{\partial \rho}{\partial t}+\nabla_{x} \cdot(v \rho)=0 \\
\frac{\partial \pi}{\partial t}+\nabla_{x} \cdot(v \pi)=\mathcal{P}(\pi, \sigma, \nu), \\
\frac{\partial \sigma}{\partial t}+\nabla_{x} \cdot(u \sigma)+\frac{\partial}{\partial m}\left[s_{l}\left(\pi-\pi_{l}(T)\right) \sigma\right]=\mathcal{S}(\pi, \sigma, \nu), \\
\frac{\partial \nu}{\partial t}+\nabla_{x} \cdot(w \nu)+\frac{\partial}{\partial m}\left[s_{s}\left(\pi-\pi_{s}(T)\right) \nu\right]=\mathcal{N}(\pi, \sigma, \nu),
\end{array}\right.
$$

where $t \geq 0, x \in \Omega$, and $m>0$;

(2.1) $v(t, x)$, the velocity of the air, is a given vector function; as for $u$ and $w$, the velocities of droplets and of ice particles, we suppose a dependence on $t, x$ and $m$; this one may well include some link with $v$, as we show in 7.1 below;

(2.2) functions $s_{l}(m)$ and $s_{s}(m)$ are proportional to the surfaces of droplets and ice particles respectively of mass $m$ : since we suppose that they always contain an aerosol kernel with mass in the interval $\left[m_{a}, M_{a}\right]$, we assume $s_{l}(m)=s_{s}(m)=0$ for every $m \leq m_{a}$; as we are not interested in too big drops or ice particles, we also feel we can assume that $s_{l}$ and $s_{s}$ are bounded;

moreover,

$$
\mathcal{P}(\pi, \sigma, \nu)(t, x)=\left[-F_{l}\left(\left[\pi-\pi_{l}(T)\right] \sigma\right)-\left(\pi-\pi_{s}(T)\right) F_{s}(\nu)\right](t, x),
$$

where $\pi_{l}$ and $\pi_{s}$ are the densities of the saturated vapor relative to the liquid and solid states; they are supposed to depend on $(t, x)$ through the temperature $T$; we also suppose that $\pi_{l}$ depends on $m$ (in [19] it did not), since transition from gaseous to liquid state is harder when $m$ is very small,

$$
\begin{aligned}
& F_{l}(\omega)(t, x)=\int_{0}^{\infty} \frac{s_{l}(m)}{m} \omega(t, x, m) d m, \quad \omega=\left(\pi-\pi_{l}\right) \sigma \\
& F_{s}(\nu)(t, x)=\int_{0}^{\infty} \frac{s_{s}(m)}{m} \nu(t, x, m) d m
\end{aligned}
$$

also,

$$
\begin{aligned}
& \mathcal{S}(\pi, \sigma, \nu)(t, x, m)= \\
& \quad=\left[\mathcal{S}_{g}(\pi, \sigma)+\mathcal{S}_{s}(\sigma, \nu)+\mathcal{S}_{a}(\pi, \sigma, \nu)+\mathcal{S}_{q}(\sigma, \nu)\right](t, x, m)
\end{aligned}
$$

where

$$
\mathcal{S}_{g}(\pi, \sigma)(t, x, m)=\frac{s_{l}(m)}{m}\left[\pi(t, x)-\pi_{l}(m, T(t, x))\right] \sigma(t, x, m)
$$

regards the phase transition between gaseous and liquid states,

$$
\mathcal{S}_{s}(\sigma, \nu)(t, x, m)=\left[-K_{l s}(m, T) \sigma(m)+K_{s l}(m, T) \nu(m)\right](t, x)
$$

regards the phase transition between solid and liquid states: $K_{l s}$ and $K_{s l}$ depend on $m$, and, through the temperature $T$, on $(t, x)$ (we suppose: $K_{l s}=0$ if $T \geq T_{0}, K_{s l}=0$ if $T \leq T_{0}$, where $T_{0}$ is the temperature of phase transition between solid and liquid states, $T_{0}$ being a smooth function of $m$ ); 


$$
\begin{aligned}
\mathcal{S}_{a}(\pi, \sigma, \nu)(t, x, m)= & \\
=g_{a}(m)\left[N^{*}(t, x)-\right. & N(\sigma, \nu)(t, x)]^{+}\left[\pi(t, x)-\pi_{l}(m, T(t, x))\right]^{+}- \\
& -g_{l}(m)\left[\pi(t, x)-\pi_{l}(m, T(t, x))\right]^{-} \sigma(t, x, m)
\end{aligned}
$$

regards the probability of the appearance and disappearance of droplets around aerosol particles: $N^{*}(t, x)$ is the number of aerosol particles per unit volume centered at $x$ at time $t$, whereas $N(\sigma, \nu)(t, x)$ is the number, per unit volume centered at $x$ at time $t$, of aerosol particles which are already inside some droplets or ice particles: we suppose

$$
\begin{aligned}
& N(\sigma, \nu)=\int_{0}^{\infty} \frac{\sigma(m)}{m} d m+\int_{0}^{\infty} \frac{\nu(m)}{m} d m+C_{l} \int_{0}^{\infty} \sigma(m) d m+ \\
& +C_{s} \int_{0}^{\infty} \nu(m) d m ;
\end{aligned}
$$

here $C_{l}$ e $C_{s}$ are constants, whereas $g_{a}$ and $g_{l}$ are given functions of $m$, related to the probability of the appearance and disappearance of droplets;

(2.11) $\mathcal{S}_{q}(\sigma, \nu)=Q_{l}(\sigma, \sigma)+J_{l}(\sigma) \sigma+J_{l s}(\nu) \sigma$

is an integral quadratic term regarding encounters of droplets with each other or with ice particles:

$$
\begin{aligned}
& J_{l}(\sigma)(t, x, m)=-m \int_{0}^{\infty} \beta_{l}\left(m, m^{\prime}\right) \sigma\left(t, x, m^{\prime}\right) d m^{\prime} \\
& Q_{l}\left(\sigma, \sigma^{\prime}\right)(t, x, m)=\frac{m}{2} \int_{0}^{m} \beta_{l}\left(m^{\prime}, m-m^{\prime}\right) \sigma\left(t, x, m^{\prime}\right) \sigma^{\prime}\left(t, x, m-m^{\prime}\right) d m^{\prime} \\
& J_{l s}(\omega)(t, x, m)=-m \int_{0}^{\infty} Z_{l s}\left(m^{\prime}, m\right) \omega\left(t, x, m^{\prime}\right) d m^{\prime}, \quad \omega=\nu, \sigma
\end{aligned}
$$

where $\beta_{l}\left(m, m^{\prime}\right)$ is related to the probability that droplets of mass $m$ and $m^{\prime}$ collide and merge, whereas $Z_{l s}\left(m, m^{\prime}\right)$ regards the probability that a droplet of mass $m^{\prime}$ joins an ice particle of mass $m$ (with phase transition from the liquid to the solid state);

further,

$$
\begin{aligned}
& \mathcal{N}(\pi, \sigma, \nu)(t, x, m)= \\
& \quad=\left[\mathcal{N}_{g}(\pi, \nu)+\mathcal{N}_{l}(\sigma, \nu)+\mathcal{N}_{a}(\pi, \nu)+\mathcal{N}_{q}(\sigma, \nu)\right](t, x, m)
\end{aligned}
$$

where

$$
\mathcal{N}_{g}(\pi, \nu)(t, x, m)=\frac{s_{s}(m)}{m}\left[\pi(t, x)-\pi_{s}(T(t, x))\right] \nu(t, x, m)
$$

regards the phase transition between gaseous and solid states,

(2.17) $\mathcal{N}_{l}(\sigma, \nu)(t, x, m)=\left[K_{l s}(m, T) \sigma(m)-K_{s l}(m, T) \nu(m)\right](t, x)$

regards the phase transition between solid and liquid states,

(2.18) $\mathcal{N}_{a}(\pi, \nu)(t, x, m)=-g_{s}(m)\left[\pi(t, x)-\pi_{s}(T(t, x))\right]^{-} \nu(t, x, m)$

regards the probability of disappearance of ice around aerosol particles,

(2.19) $\mathcal{N}_{q}(\sigma, \nu)=Q_{s}(\nu, \nu)+J_{s}(\nu) \nu+Q_{l s}(\nu, \sigma)+J_{l s}(\sigma) \nu$

is an integral quadratic term regarding encounters of ice particles with each other or with droplets, where (see also (2.14)): 


$$
J_{s}(\nu)(t, x, m)=-m \int_{0}^{\infty} \beta_{s}\left(m, m^{\prime}\right) \nu\left(t, x, m^{\prime}\right) d m^{\prime}
$$

$$
Q_{s}\left(\nu, \nu^{\prime}\right)(t, x, m)=\frac{m}{2} \int_{0}^{m} \beta_{s}\left(m-m^{\prime}, m^{\prime}\right) \nu\left(t, x, m^{\prime}\right) \nu^{\prime}\left(t, x, m-m^{\prime}\right) d m^{\prime}
$$

$$
Q_{l s}(\nu, \sigma)(t, x, m)=m \int_{0}^{m} Z_{l s}\left(m-m^{\prime}, m^{\prime}\right) \nu\left(t, x, m-m^{\prime}\right) \sigma\left(t, x, m^{\prime}\right) d m^{\prime}
$$

where $\beta_{s}\left(m, m^{\prime}\right)$ is related to the probability that ice particles of masses $m$ and $m^{\prime}$ collide and merge.

(2.23) In accordance with the physical model proposed in [19], we assume that each of the functions $s_{l}(m), \quad s_{s}(m), \quad K_{l s}(m, T), \quad K_{s l}(m, T)$, $N^{*}(t, x), \quad g_{a}(m), \quad g_{l}(m), \quad g_{s}(m), \quad \beta_{l}\left(m, m^{\prime}\right), \quad \beta_{s}\left(m, m^{\prime}\right), \quad Z_{l s}\left(m, m^{\prime}\right)$, $\pi_{l}(m, T)$ and $\pi_{s}(T)$ are non-negative. We also assume that they, as well as $\partial_{m} \pi_{l}, s_{l}^{\prime}$ and $s_{s}^{\prime}$, are bounded and Lipschitz continuous.

As for $g_{a}(m)$, we suppose that

(2.24) $\operatorname{supp} g_{a}(\cdot) \subseteq\left[m_{a}, M_{a}\right]$.

Since it does not make sense to consider too big droplets or ice particles, we also feel we can assume that, for some $M>M_{a}$,

$$
\beta_{l}\left(m^{\prime}, m^{\prime \prime}\right)=\beta_{s}\left(m^{\prime}, m^{\prime \prime}\right)=Z_{l s}\left(m^{\prime}, m^{\prime \prime}\right)=0
$$

whenever $m^{\prime}+m^{\prime \prime} \geq M$.

\section{The main result}

We suppose now that the bounded open set $\Omega$ of $\mathbf{R}^{3}$ has a strongly Lipschitz continuous boundary, and define $\left.\Omega^{+}=\Omega \times\right] 0,+\infty[$; the first two equations of system (Q) hold on $\left.Q_{\tau}=\right] 0, \tau[\times \Omega$, whereas the other ones hold on $\left.S_{\tau}=\right] 0, \tau\left[\times \Omega^{+}\right.$.

Let $q \in[1, \infty]$, and let $q^{\prime}$ be its conjugate exponent. We make the following assumptions on temperature and velocities:

$$
\begin{aligned}
& T \in L^{q}(] 0, \tau\left[; W^{1, \infty}(\Omega)\right), \\
& v \in L^{q}(] 0, \tau\left[; W^{1, \infty}(\Omega)\right)^{3}, \quad \nabla \cdot v \in L^{q}(] 0, \tau\left[; W^{1, \infty}(\Omega)\right), \\
& u \in L^{q}(] 0, \tau\left[; W^{1, \infty}\left(\Omega^{+}\right)\right)^{3}, \quad \nabla \cdot u \in L^{q}(] 0, \tau\left[; W^{1, \infty}\left(\Omega^{+}\right)\right), \\
& w \in L^{q}(] 0, \tau\left[; W^{1, \infty}\left(\Omega^{+}\right)\right)^{3}, \quad \nabla \cdot w \in L^{q}(] 0, \tau\left[; W^{1, \infty}\left(\Omega^{+}\right)\right) .
\end{aligned}
$$

For a brief discussion of the dependence of $u$ and $w$ on $v$ see 7.1 below. We also assume that the velocities satisfy the boundary conditions ( $n$ is the exterior normal unit vector that is defined a. e. at the boundary of $\Omega$ ):

$$
\left.n \cdot v\right|_{\partial \Omega}=\left.n \cdot u\right|_{\partial \Omega}=\left.n \cdot w\right|_{\partial \Omega}=0 .
$$

As for initial conditions, we assume: 


$$
\begin{array}{ll}
\rho(0, \cdot)=\rho_{0} \in W^{1, \infty}(\Omega), & \rho_{0} \geq 0 \\
\pi(0, \cdot)=\pi_{0} \in W^{1, \infty}(\Omega), & \pi_{0} \geq 0 \\
\sigma(0, \cdot)=\sigma_{0} \in W^{1, \infty}\left(\Omega^{+}\right), & \sigma_{0} \geq 0 \\
\nu(0, \cdot)=\nu_{0} \in W^{1, \infty}\left(\Omega^{+}\right), & \nu_{0} \geq 0 \\
\sigma_{0}(x, m)=\nu_{0}(x, m)=0 & \text { if } \left.\quad m \in] 0, m_{a}\right] \cup[M,+\infty[
\end{array}
$$

We can now present the main result of this work.

Theorem 3.1. Assume all hypotheses stated above on all functions involved in the equations of system $(Q)$. Then there exists $\left.\left.\tau^{*} \in\right] 0, \tau\right]$ such that the Cauchy problem for system $(Q)$, with initial data as in (3.6)-(3.10), has, in the time interval $\left[0, \tau^{*}\right]$, one weak solution $(\rho, \pi, \sigma, \nu)$ such that

(3.11) $\rho, \pi \in L^{\infty}(] 0, \tau^{*}\left[; W^{1, \infty}(\Omega)\right) \cap W^{1, q}(] 0, \tau^{*}\left[: L^{\infty}(\Omega)\right)$,

(3.12) $\sigma, \nu \in L^{\infty}(] 0, \tau^{*}\left[; W^{1, \infty}\left(\Omega^{+}\right)\right) \cap W^{1, q}(] 0, \tau^{*}\left[; L^{\infty}\left(\Omega^{+}\right)\right)$;

(3.13) $\rho, \pi, \sigma, \nu$ take non-negative values, $\rho$ satisfies the estimate

(3.14) $\inf _{x \in \Omega} \rho(t) \geq e^{-\int_{0}^{t}\left\|\nabla \cdot v\left(t^{\prime}\right)\right\|_{\infty, \Omega} d t^{\prime}} \inf _{x \in \Omega} \rho_{0}$,

and further

(3.15) $\operatorname{supp} \sigma(t, x, \cdot), \operatorname{supp} \nu(t, x, \cdot) \subseteq\left[m_{a}, M\right] \quad \forall(t, x) \in\left[0, \tau^{*}\right] \times \Omega$.

Moreover, this weak solution is unique in the space $L^{\infty}(] 0, \tau^{*}\left[; W^{1, \infty}(\Omega)\right)^{2}$ $\times L^{\infty}(] 0, \tau^{*}\left[; W^{1, \infty}\left(\Omega^{+}\right)\right)^{2}$.

Furthermore, for any sufficiently small t, the mapping

$\left(\rho_{0}, \pi_{0}, \sigma_{0}, \nu_{0}, T, v, u, w, \nabla \cdot v, \nabla_{x} \cdot u, \nabla_{x} \cdot w\right) \mapsto(\rho, \pi, \sigma, \nu)$

is a locally Lipschitz continuous function from the space $W^{1, \infty}(\Omega)^{2} \times$ $W^{1, \infty}\left(\Omega^{+}\right)^{2} \times L^{q}(] 0, t\left[; L^{\infty}(\Omega)\right)^{4} \times L^{q}(] 0, t\left[; L^{\infty}\left(\Omega^{+}\right)\right)^{6} \times L^{q}(] 0, t\left[; L^{\infty}(\Omega)\right) \times$ $L^{q}(] 0, t\left[; L^{\infty}\left(\Omega^{+}\right)\right)^{2}$ to the space $L^{\infty}\left(Q_{t}\right)^{2} \times L^{\infty}\left(S_{t}\right)^{2}$.

\section{Preliminary results on linear transport and continuity equations}

Let $\Omega$ be a bounded open set of $\mathbf{R}^{d}, \partial \Omega$ its boundary, which we assume to be strongly Lipschitz continuous, so that an exterior normal unit vector $n$ is defined a.e. on it, $\left.Q_{\tau}=\right] 0, \tau[\times \Omega$.

Let us consider the Cauchy problem for the linear transport equation on $Q_{\tau}$

(4.1) $\frac{\partial z}{\partial t}(t, x)+b(t, x) \cdot \nabla z(t, x)+c(t, x) z(t, x)=f(t, x)$,

with initial datum on $\Omega$

(4.2) $z(\cdot, 0)=z_{0}$.

Let $b \in L^{1}\left(Q_{\tau}\right)^{d}, \nabla \cdot b-c, f \in L^{1}\left(Q_{\tau}\right)$ and $z_{0} \in L^{\infty}(\Omega)$. By a weak solution of the Cauchy problem (4.1)-(4.2) we mean a function $z \in L^{\infty}\left(Q_{\tau}\right)$ such that 


$$
\int_{0}^{\tau} d t \int_{\Omega}\left[z\left(\frac{\partial \varphi}{\partial t}+b \cdot \nabla \varphi+(\nabla \cdot b) \varphi-c \varphi\right)+f \varphi\right] d x=-\int_{\Omega} z_{0} \varphi(0, \cdot) d x
$$

$\forall \varphi \in C_{c}^{0,1}\left(\left[0, \tau[\times \Omega) \quad\right.\right.$ (i.e. $\forall \varphi \in C^{0,1}([0, \tau[\times \Omega)$ whose support is compact in $\left[0, \tau[\times \Omega)\right.$, or equivalently - by density, since $L^{\infty}(] 0, \tau\left[; W^{1, \infty}(\Omega)\right)$ $\cap W^{1,1}(] 0, \tau\left[; L^{\infty}(\Omega)\right)$ is contained in $W^{1,1}(] 0, \tau[\times \Omega)$ - for every $\varphi$ in $L^{\infty}(] 0, \tau\left[; W^{1, \infty}(\Omega)\right) \cap W^{1,1}(] 0, \tau\left[; L^{\infty}(\Omega)\right)$ with compact support in $[0, \tau[\times \Omega$.

Lemma 4.1. (i) If $b \in L^{1}\left(Q_{\tau}\right)^{d}, \quad \nabla \cdot b \in L^{1}(] 0, \tau\left[; L^{\infty}(\Omega)\right), \quad c, f \in$ $L^{1}(] 0, \tau\left[; L^{\infty}(\Omega)\right), n \cdot b=0$ a.e. on $\partial \Omega$ for almost every $\left.t \in\right] 0, \tau[$, and $z_{0} \in W^{1, \infty}(\Omega)$, then:

(a) $z \in L^{\infty}(] 0, \tau\left[; W^{1, \infty}(\Omega)\right) \cap W^{1,1}(] 0, \tau\left[; L^{\infty}(\Omega)\right)$ is a weak solution if and only if it satisfies (4.1) and (4.2) almost everywhere in $Q_{\tau}$ and in $\Omega$ respectively;

(b) a weak solution $z$ lying in $L^{\infty}(] 0, \tau\left[; W^{1, \infty}(\Omega)\right) \cap W^{1,1}(] 0, \tau\left[; L^{\infty}(\Omega)\right)$ satisfies the estimate $\|z(t)\|_{\infty, \Omega} \leq e^{\int_{0}^{t}\left\|c\left(t^{\prime}\right)\right\|_{\infty, \Omega} d t^{\prime}}\left(\left\|z_{0}\right\|_{\infty, \Omega}+\int_{0}^{t}\left\|f\left(t^{\prime}\right)\right\|_{\infty, \Omega} d t^{\prime}\right)$

(c) there exists at most one weak solution $z$ of (4.1)-(4.2) lying in $L^{\infty}(] 0, \tau\left[; W^{1, \infty}(\Omega)\right) \cap W^{1,1}(] 0, \tau\left[; L^{\infty}(\Omega)\right)$.

(ii) In the hypotheses of (i), let moreover $z$ and $z^{\prime}$ be weak solutions in $L^{\infty}(] 0, \tau\left[; W^{1, \infty}(\Omega)\right) \cap W^{1,1}(] 0, \tau\left[; L^{\infty}(\Omega)\right)$, the former of (4.1)-(4.2), the latter of the perturbed Cauchy problem with initial value $z_{0}^{\prime} \in W^{1, \infty}(\Omega)$ for the equation

$$
\frac{\partial z^{\prime}}{\partial t}(t, x)+b^{\prime}(t, x) \cdot \nabla z^{\prime}(t, x)+c^{\prime}(t, x) z^{\prime}(t, x)=f^{\prime}(t, x)
$$

where $b^{\prime}, c^{\prime}$ and $f^{\prime}$ satisfy the same hypotheses as $b, c$ and $f$ in (i); then, for every $t \in[0, \tau]$, the following perturbation estimate holds:

$$
\begin{aligned}
&\left\|z(t)-z^{\prime}(t)\right\|_{\infty, \Omega} \leq e^{\int_{0}^{t}\left\|c\left(t^{\prime}\right)\right\|_{\infty, \Omega} d t^{\prime}}\left(\left\|z_{0}-z_{0}^{\prime}\right\|_{\infty, \Omega}+\right. \\
&+\int_{0}^{t}\left(\left\|\nabla z^{\prime}\left(t^{\prime}\right)\right\|_{\infty, \Omega}\left\|b\left(t^{\prime}\right)-b^{\prime}\left(t^{\prime}\right)\right\|_{\infty, \Omega}+\right. \\
&\left.\left.+\left\|z^{\prime}\left(t^{\prime}\right)\right\|_{\infty, \Omega}\left\|c\left(t^{\prime}\right)-c^{\prime}\left(t^{\prime}\right)\right\|_{\infty, \Omega}+\left\|f\left(t^{\prime}\right)-f^{\prime}\left(t^{\prime}\right)\right\|_{\infty, \Omega}\right) d t^{\prime}\right) .
\end{aligned}
$$

Proof. (i-a) can be easily verified making use of integration by parts. (i-b): integration by parts yields, $\forall p \in[2, \infty[$,

$$
\int_{\Omega}(b \cdot \nabla z)|z|^{p-2} z=-\frac{1}{p} \int_{\Omega}(\nabla \cdot b)|z|^{p} ;
$$

after multiplying equation (4.1) by $|z|^{p-2} z$ one then gets

$$
\frac{1}{p} \frac{d}{d t} \int_{\Omega}|z|^{p} d x=\int_{\Omega}\left[\frac{1}{p}(\nabla \cdot b) z-c z+f\right]|z|^{p-2} z d x,
$$


from which, by Hölder inequality, it follows

$$
\begin{aligned}
& \frac{d}{d t}\|z(t)\|_{p, \Omega}=\int_{\Omega}\left[\frac{1}{p}(\nabla \cdot b) z-c z+f\right] \frac{|z|^{p-2} z}{\|z\|_{p, \Omega}^{p-1}} d x \leq \\
& \leq\left\|\frac{1}{p} \nabla \cdot b(t)-c(t)\right\|_{\infty, \Omega}\|z(t)\|_{p, \Omega}+\|f(t)\|_{p, \Omega} ;
\end{aligned}
$$

applying Gronwall's lemma, one gets

$$
\|z(t)\|_{p, \Omega} \leq e^{\int_{0}^{t}\left\|\frac{1}{p} \nabla \cdot b\left(t^{\prime}\right)-c\left(t^{\prime}\right)\right\|_{\infty, \Omega} d t^{\prime}}\left(\left\|z_{0}\right\|_{p, \Omega}+\int_{0}^{t}\left\|f\left(t^{\prime}\right)\right\|_{p, \Omega} d t^{\prime}\right)
$$

taking the limit as $p \rightarrow \infty$, since $\|f(t)\|_{p, \Omega} \leq\|f(t)\|_{\infty, \Omega}|\Omega|^{1 / p}$ a.e. on $] 0, \tau[$, by Lebesgue's theorem estimate (4.4) follows.

(i-c): the uniqueness of $z$ follows from estimate (4.4), since it is equivalent to the fact that if $f=0$ and $z_{0}=0$ then also $z=0$.

(ii): $z-z^{\prime}$ satisfies the equation

$$
\begin{aligned}
\frac{\partial}{\partial t}\left(z-z^{\prime}\right) & +b \cdot \nabla\left(z-z^{\prime}\right)+c\left(z-z^{\prime}\right)= \\
& =\left(f-f^{\prime}\right)-\left(b-b^{\prime}\right) \cdot \nabla z^{\prime}-\left(c-c^{\prime}\right) z^{\prime}
\end{aligned}
$$

applying (i-b) to $z-z^{\prime}$ yields (4.5).

Lemma 4.2. Under the weaker hypotheses (with respect to Lemma 4.1) that $b \in L^{1}\left(Q_{\tau}\right)^{d}, \nabla \cdot b \in L^{1}\left(Q_{\tau}\right), c, f \in L^{1}(] 0, \tau\left[; L^{\infty}(\Omega)\right), z_{0} \in L^{\infty}(\Omega)$, and that, for almost every $t \in] 0, \tau[, n \cdot b=0$ a. e. on $\partial \Omega$, then there exists a weak solution $z$ of the Cauchy problem (4.1)-(4.2) satisfying, for almost every $t \in[0, \tau]$, estimate (4.4); moreover, if $f$ and $z_{0}$ are non-negative, such a solution $z$ is non-negative too.

Proof. We can extend $b, c, f$, and respectively $z_{0}$, to functions with compact support in $\mathbf{R} \times \mathbf{R}^{d}$, and respectively in $\mathbf{R}^{d}$, so as to get $b \in L^{1}\left(\mathbf{R} ; L^{1}\left(\mathbf{R}^{d}\right)\right)^{d}$, $\nabla \cdot b \in L^{1}\left(\mathbf{R} ; L^{1}\left(\mathbf{R}^{d}\right)\right), c, f \in L^{1}\left(\mathbf{R} ; L^{\infty}\left(\mathbf{R}^{d}\right)\right), z_{0} \in L^{\infty}\left(\mathbf{R}^{d}\right)$. Insofar as we are not interested in the regularity of $z$, we may even choose $b=0, c=f=0$ and $z_{0}=0$ outside $\Omega$.

Let then $\theta_{k}(t)$ and $\xi_{k}(x)$ be regularizing sequences satisfying the usual properties, and endowed with a support of radius $1 / k$; and let $\eta_{k}(t, x)=$ $\theta_{k}(t) \xi_{k}(x)$. Let $b_{k}=b * \eta_{k}, c_{k}=c * \eta_{k}, f_{k}=f * \eta_{k}, z_{0}^{k}=z_{0} * \xi_{k}$; they all have compact support.

Let $z^{k}$ be the solution of the Cauchy problem in $\mathbf{R} \times \mathbf{R}^{d}$ for the equation

$$
\frac{\partial z^{k}}{\partial t}(t, x)+b_{k}(t, x) \cdot \nabla z^{k}(t, x)+c_{k}(t, x) z^{k}(t, x)=f_{k}(t, x),
$$

with initial datum on $\mathbf{R}^{d}$

$$
z^{k}(\cdot, 0)=z_{0}^{k}
$$

$z^{k}$ can be obtained e.g. by integrating along characteristics, so that, if $f$ and $z_{0}$ are non-negative, also $z^{k}$ is, and it satisfies, by Lemma 4.1(i-b), the estimate

$$
\left\|z^{k}(t)\right\|_{\infty, \mathbf{R}^{d}} \leq e^{\int_{0}^{t}\left\|c_{k}\left(t^{\prime}\right)\right\|_{\infty, \mathbf{R}^{d}} d t^{\prime}}\left(\left\|z_{0}^{k}\right\|_{\infty, \mathbf{R}^{d}}+\int_{0}^{t}\left\|f_{k}\left(t^{\prime}\right)\right\|_{\infty, \mathbf{R}^{d}} d t^{\prime}\right),
$$


from which it follows, by Young's inequality,

$$
\left\|z^{k}(t)\right\|_{\infty, \mathbf{R}^{d}} \leq e^{\int_{-1 / k}^{t+1 / k}\left\|c\left(t^{\prime}\right)\right\|_{\infty, \mathbf{R}^{d}} d t^{\prime}}\left(\left\|z_{0}\right\|_{\infty, \mathbf{R}^{d}}+\int_{-1 / k}^{t+1 / k}\left\|f\left(t^{\prime}\right)\right\|_{\infty, \mathbf{R}^{d}} d t^{\prime}\right)
$$

(one has indeed, for example,

$$
\begin{aligned}
& \int_{0}^{t}\left\|c_{k}\left(t^{\prime}\right)\right\|_{\infty, \mathbf{R}^{d}} d t^{\prime} \leq \int_{0}^{t}\left\|\int_{\mathbf{R}} \theta_{k}\left(t^{\prime \prime}\right) c\left(t^{\prime}-t^{\prime \prime}\right) d t^{\prime \prime}\right\|_{\infty, \mathbf{R}^{d}} d t^{\prime} \leq \\
& \leq \int_{0}^{t} \int_{-1 / k}^{1 / k} \theta_{k}\left(t^{\prime \prime}\right)\left\|c\left(t^{\prime}-t^{\prime \prime}\right)\right\|_{\infty, \mathbf{R}^{d}} d t^{\prime \prime} d t^{\prime} \\
& \left.\leq \int_{-1 / k}^{1 / k} \theta_{k}\left(t^{\prime \prime}\right) \int_{-1 / k}^{t+1 / k}\left\|c\left(t^{\prime}\right)\right\|_{\infty, \mathbf{R}^{d}} d t^{\prime} d t^{\prime \prime}\right) .
\end{aligned}
$$

Hence, there exists a subsequence $z^{k_{j}}$ of $z^{k}$, that converges weakly* in $L^{\infty}(] 0, \tau\left[; L^{\infty}\left(\mathbf{R}^{d}\right)\right)$, as $j \rightarrow \infty$, to a function $z$ satisfying (4.4) (referred to $\mathbf{R}^{d}$ or to $\Omega)$; as a consequence, if $f$ and $z_{0}$, and hence every $z^{k}$, are non-negative, also $z$ is non-negative. Since moreover $z^{k}$ is a weak solution of the Cauchy problem (4.8)-(4.9), one has, $\forall \varphi \in L^{\infty}(] 0, \tau\left[; W^{1, \infty}(\Omega)\right) \cap W^{1,1}(] 0, \tau\left[; L^{\infty}(\Omega)\right)$ with compact support in $[0, \tau[\times \Omega$,

$$
\begin{aligned}
& \int_{0}^{\tau} d t \int_{\mathbf{R}^{d}}\left[z^{k}\left(\frac{\partial \varphi}{\partial t}+b_{k} \cdot \nabla \varphi+\left(\nabla \cdot b_{k}\right) \varphi-c_{k} \varphi\right)+f_{k} \varphi\right] d x= \\
= & -\int_{\mathbf{R}^{d}} z_{0}^{k} \varphi(0, \cdot) d x
\end{aligned}
$$

since, as $k \rightarrow \infty, b_{k}, \nabla \cdot b_{k}, c_{k}, f_{k}$ and $z_{0}^{k}$ tend strongly in the $L^{1}$ norm to $b, \nabla \cdot b, c, f$ and $z_{0}$ respectively, it follows that $z$ satisfies (4.3) (even referred to $\left.\mathbf{R}^{d}\right)$, that is $z$ is a weak solution. Note that if $z \in L^{\infty}(] 0, \tau\left[; W^{1, \infty}\left(\mathbf{R}^{d}\right)\right) \cap$ $W^{1,1}(] 0, \tau\left[; L^{\infty}\left(\mathbf{R}^{d}\right)\right)$ and $\nabla \cdot b \in L^{1}(] 0, \tau\left[; L^{\infty}\left(\mathbf{R}^{d}\right)\right)$, by applying (4.5) to $z^{k}-z$ one finds that $z^{k} \rightarrow z$ strongly in $L^{\infty}(] 0, \tau\left[\times \mathbf{R}^{d}\right)$.

Lemma 4.3. Let $\nu \in L^{1}\left([0, \tau] ; \mathbf{R}^{+}\right)$satisfy for almost every $t_{0}$ and $t$ in $] 0, \tau[$ with $t_{0}<t$, and in particular for $t_{0}=0$, the estimate

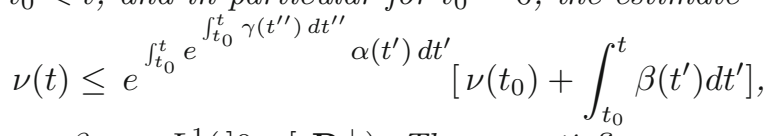

where $\alpha, \beta, \gamma \in L^{1}(] 0, \tau\left[; \mathbf{R}^{+}\right)$. Then $\nu$ satisfies

$$
\nu(t) \leq e^{\int_{0}^{t} \alpha\left(t^{\prime}\right) d t^{\prime}}\left[\nu(0)+\int_{0}^{t} \beta\left(t^{\prime}\right) d t^{\prime}\right]
$$

Proof. Choose intervals $I_{j}^{k}$ with $k>0, j=1, \ldots, k$, where $I_{j}^{k}=\left[t_{j-1}^{k}, t_{j}^{k}\right]$ is such that the given estimate holds with $t_{0}=t_{j-1}^{k}, t=t_{j}^{k}$, and moreover, for $j=0, \ldots, k, t_{j}^{k}$ is such that $t_{0}^{k}=0, t_{k}^{k}=t$, and $\max _{j=1, \ldots, k}\left(t_{j}^{k}-t_{j-1}^{k}\right) \rightarrow 0$ as $k \rightarrow \infty$; one then gets, iterating the given estimate on $I_{j}^{k}$ for $j=1, \ldots, k$, 
$\nu(t) \leq e^{\sum_{j=1}^{k} \int_{I_{j}^{k}} e^{\int_{I_{j}^{k}} \gamma\left(t^{\prime \prime}\right) d t^{\prime \prime}} \alpha\left(t^{\prime}\right) d t^{\prime}} \nu(0)+\sum_{j=1}^{k}\left[e^{\sum_{i=1}^{k} \int_{I_{i}^{k}} e^{\int_{I_{i}^{k}} \gamma\left(t^{\prime \prime}\right) d t^{\prime \prime}} \alpha\left(t^{\prime}\right) d t^{\prime}} \int_{I_{j}^{k}} \beta\left(t^{\prime}\right) d t^{\prime}\right] ;$ letting then $k \rightarrow \infty$, since $\int_{I_{j}^{k}} \varphi_{j}^{k}(t) d t=\int_{0}^{t} \chi_{j}^{k}\left(t^{\prime}\right) \varphi_{j}^{k}\left(t^{\prime}\right) d t^{\prime}$, where $\chi_{j}^{k}$ is the characteristic function of $I_{j}^{k}$ and $\varphi_{j}^{k}(t)=\alpha(t) \exp \int_{I_{j}^{k}} \gamma\left(t^{\prime}\right) d t^{\prime}$, by Lebesgue's theorem the result follows.

Lemma 4.4. Under the stronger hypotheses - with respect to Lemma 4.1-that $b \in L^{1}(] 0, \tau\left[; W^{1, \infty}(\Omega)\right)^{d}, c, f \in L^{1}(] 0, \tau\left[; W^{1, \infty}(\Omega)\right), n \cdot b=0$ a.e. on $\partial \Omega$ for almost every $t \in] 0, \tau\left[\right.$, and $z_{0} \in W^{1, \infty}(\Omega)$, then:

(a) a weak solution $z$ lying in $L^{\infty}(] 0, \tau\left[; W^{1, \infty}(\Omega)\right) \cap W^{1,1}(] 0, \tau\left[; L^{\infty}(\Omega)\right)$ satisfies, in addition to estimate (4.4), for almost every $t \in[0, \tau]$, also the estimate

$$
\begin{aligned}
& \|\nabla z(t)\|_{\infty, \Omega} \leq e^{\int_{0}^{t}\left(\left\|D b\left(t^{\prime}\right)\right\|_{\infty, \Omega}+\left\|c\left(t^{\prime}\right)\right\|_{\infty, \Omega}\right) d t^{\prime}}\left(\left\|\nabla z_{0}\right\|_{\infty, \Omega}+\right. \\
& \left.+\int_{0}^{t}\left(\left\|z\left(t^{\prime}\right)\right\|_{\infty, \Omega}\left\|\nabla c\left(t^{\prime}\right)\right\|_{\infty, \Omega}+\left\|\nabla f\left(t^{\prime}\right)\right\|_{\infty, \Omega}\right) d t^{\prime}\right)
\end{aligned}
$$

where $D b$ denotes the jacobian matrix of $b$ with respect to $x$, and $\mathbf{R}^{d}$ is endowed with the maximum norm;

(b) such a solution does exist; namely, if for some $q \in[1, \infty] b$ also lies in $L^{q}(] 0, \tau\left[; L^{\infty}(\Omega)\right)^{d}$ and $c$ and $f$ lie also in $L^{q}(] 0, \tau\left[; L^{\infty}(\Omega)\right)$, then $z$ lies in $L^{\infty}(] 0, \tau\left[; W^{1, \infty}(\Omega)\right) \cap W^{1, q}(] 0, \tau\left[; L^{\infty}(\Omega)\right)$; in case $q=\infty$, note that $L^{\infty}(] 0, \tau\left[; W^{1, \infty}(\Omega)\right) \cap W^{1, \infty}(] 0, \tau\left[; L^{\infty}(\Omega)\right)=W^{1, \infty}\left(Q_{\tau}\right) ;$

(c) if, further, $\nabla \cdot b \in L^{1}(] 0, \tau\left[; W^{1, \infty}(\Omega)\right)$, then $z$ is unique in the wider class of weak solutions of problem (4.1)-(4.2).

Proof. (a): suppose first that $\partial_{i} z=\frac{\partial z}{\partial x_{i}}$ is a weak solution $w_{i}$ of the equation

$$
\frac{\partial w_{i}}{\partial t}+b \cdot \nabla w_{i}+c w_{i}=\partial_{i} f-\left(\partial_{i} b\right) \cdot \nabla z-\left(\partial_{i} c\right) z
$$

with initial datum $\partial_{i} z_{0}$ and satisfying estimate (4.4) for almost every $t$ (it exists by Lemma 4.2), which yields:

$$
\begin{aligned}
& \left\|\partial_{i} z(t)\right\|_{\infty, \Omega} \leq e^{\int_{0}^{t}\left\|c\left(t^{\prime}\right)\right\|_{\infty, \Omega} d t^{\prime}}\left(\left\|\partial_{i} z_{0}\right\|_{\infty, \Omega}+\int_{0}^{t}\left(\left\|\partial_{i} f\left(t^{\prime}\right)\right\|_{\infty, \Omega}+\right.\right. \\
& \left.\left.+\left\|\partial_{i} b\left(t^{\prime}\right)\right\|_{\infty, \Omega}\left\|\nabla z\left(t^{\prime}\right)\right\|_{\infty, \Omega}+\left\|z\left(t^{\prime}\right)\right\|_{\infty, \Omega}\left\|\partial_{i} c\left(t^{\prime}\right)\right\|_{\infty, \Omega}\right) d t^{\prime}\right) ;
\end{aligned}
$$

it follows

$$
\begin{aligned}
& \|\nabla z(t)\|_{\infty, \Omega} \leq e^{\int_{0}^{t}\left\|c\left(t^{\prime}\right)\right\|_{\infty, \Omega} d t^{\prime}}\left(\left\|\nabla z_{0}\right\|_{\infty, \Omega}+\int_{0}^{t}\left(\left\|\nabla f\left(t^{\prime}\right)\right\|_{\infty, \Omega}+\right.\right. \\
& \left.\left.+\left\|D b\left(t^{\prime}\right)\right\|_{\infty, \Omega}\left\|\nabla z\left(t^{\prime}\right)\right\|_{\infty, \Omega}+\left\|z\left(t^{\prime}\right)\right\|_{\infty, \Omega}\left\|\nabla c\left(t^{\prime}\right)\right\|_{\infty, \Omega}\right) d t^{\prime}\right),
\end{aligned}
$$


and, applying Gronwall's lemma, one gets

$$
\begin{gathered}
\|\nabla z(t)\|_{\infty, \Omega} \leq e^{\int_{0}^{t}\left(e^{\int_{0}^{t}\left\|c\left(t^{\prime \prime}\right)\right\|_{\infty, \Omega} d t^{\prime \prime}}\left\|D b\left(t^{\prime}\right)\right\|_{\infty, \Omega}+\left\|c\left(t^{\prime}\right)\right\|_{\infty, \Omega}\right) d t^{\prime}}\left(\left\|\nabla z_{0}\right\|_{\infty, \Omega}+\right. \\
\left.+\int_{0}^{t}\left(\left\|z\left(t^{\prime}\right)\right\|_{\infty, \Omega}\left\|\nabla c\left(t^{\prime}\right)\right\|_{\infty, \Omega}+\left\|\nabla f\left(t^{\prime}\right)\right\|_{\infty, \Omega}\right) d t^{\prime}\right)
\end{gathered}
$$

by Lemma 4.3, estimate (4.11) follows.

But the identity $w_{i}=\partial_{i} z$ is obvious in the case of smooth coefficients and initial datum; hence, in the general case, an estimate (4.11) may be applied in $\mathbf{R}^{d}$ to the sequence $z^{k}$ we constructed when proving Lemma 4.2 , and yields that the sequence $\nabla z^{k}$ is bounded in $L^{\infty}(] 0, \tau\left[\times \mathbf{R}^{d}\right)^{d}$; hence there exists a subsequence $\nabla z^{k_{l}}$ of $\nabla z^{k_{j}}$, converging weakly*, as $l \rightarrow \infty$, to $\nabla z$ (since $\nabla z^{k_{l}} \rightarrow \nabla z$ already in $\left.\mathcal{D}^{\prime}(] 0, \tau\left[\times \mathbf{R}^{d}\right)^{d}\right)$. From here it follows that $z \in L^{\infty}(] 0, \tau\left[; W^{1, \infty}\left(\mathbf{R}^{d}\right)\right)$. Passing to the limit in the definition of weak solution yields that the identity $w_{i}=\partial_{i} z$ holds.

(b): we have already shown that a solution $z$ exists in $L^{\infty}(] 0, \tau\left[; W^{1, \infty}\left(\mathbf{R}^{d}\right)\right) ; \quad(4.10) \quad$ readily $\quad$ yields $\quad \frac{\partial z}{\partial t}=f-b \cdot \nabla z-c z \quad \in$ $L^{q}(] 0, \tau\left[; L^{\infty}\left(\mathbf{R}^{d}\right)\right)$; hence we have also $z \in W^{1, q}(] 0, \tau\left[; L^{\infty}\left(\mathbf{R}^{d}\right)\right)$.

(c): taking the difference between two solutions, one sees that it suffices to prove that, in case (4.3) holds with $f=0$ and $z_{0}=0$, then $z=0$. In such a case, (4.3) will hold in particular for every solution of the Cauchy problem in $[0, \tau[\times \Omega$

$$
\left\{\begin{array}{l}
\frac{\partial \varphi}{\partial t}+b \cdot \nabla \varphi+(\nabla \cdot b) \varphi-c \varphi=h \\
\varphi(\tau-\varepsilon, x)=0 \quad \forall x \in \Omega,
\end{array}\right.
$$

where $h \in L^{1}(] 0, \tau\left[; W^{1, \infty}(\Omega)\right)$ vanishes in $[0, \tau-\varepsilon[\times(\Omega \backslash K) \cup[\tau-\varepsilon, \tau[\times \Omega$ for some $\varepsilon>0$ and $K \subset \Omega$ compact. By (b) (applied also with reverted time) such a solution exists in the required space, and hence

$$
\int_{0}^{\tau} d t \int_{\Omega} z(t, x) h(t, x) d x=0
$$

for every $h$ with the aforesaid properties. It follows $z=0$ a.e.

Remarks 4.5. (a) It can be easily verified that under the hypotheses of Lemma 4.2 , if moreover $b \in L^{1}(] 0, \tau\left[; L^{\infty}(\Omega)\right)^{d}$, then the weak solution we constructed in our proof satisfies also the perturbation estimate (4.5), whenever $z^{\prime}$ is as in (ii) of Lemma 4.1.

(b) $L^{p}$-estimates similar to (4.11) and to (4.5) may be analogously derived making use of estimate (4.6) instead of estimate (4.4).

(c) We do not further discuss, in this paper, the uniqueness of weak solutions; related results can be found e.g. in $[1,2,6,11]$.

Corollary 4.6. Let $\Omega$ be a bounded open subset of $\mathbf{R}^{d}$ endowed with a strongly Lipschitz continuous boundary, and let $\left.Q_{\tau}=\Omega \times\right] 0, \tau[$. Let $q \in[1, \infty]$, and let $q^{\prime}$ be its conjugate exponent. Given 


$$
b \in L^{q}(] 0, \tau\left[; W^{1, \infty}(\Omega)\right)^{d}, \quad c, f \in L^{q}(] 0, \tau\left[; W^{1, \infty}(\Omega)\right), z_{0} \in W^{1, \infty}(\Omega),
$$

with $\left.n \cdot b\right|_{\partial \Omega}=0$, then

(i) there exists in $L^{\infty}(] 0, \tau\left[; W^{1, \infty}(\Omega)\right) \cap W^{1, q}(] 0, \tau\left[; L^{\infty}(\Omega)\right)$ a unique (weak) solution $z$ of the problem

$$
\left\{\begin{array}{l}
\frac{\partial z}{\partial t}+b \cdot \nabla z+c z=f \text { in } Q_{\tau} \\
z(\cdot, 0)=z_{0}(\cdot) \text { in } \Omega
\end{array}\right.
$$

and the following estimates hold:

$$
\begin{aligned}
& \|z\|_{L^{\infty}\left(0, \tau ; W^{1, \infty}(\Omega)\right)} \leq e^{\int_{0}^{\tau}\left(\|D b(t)\|_{L^{\infty}(\Omega)}+\|c(t)\|_{W^{1, \infty}(\Omega)}\right) d t}\left(\left\|z_{0}\right\|_{W^{1, \infty}(\Omega)}+\right. \\
& \left.\quad+\tau^{1 / q^{\prime}}\|f\|_{L^{q}\left(0, \tau ; W^{1, \infty}(\Omega)\right)}\right)
\end{aligned}
$$

$$
\left\|\frac{\partial z}{\partial t}\right\|_{L^{q}\left(0, \tau ; L^{\infty}(\Omega)\right)} \leq M_{c, b}^{q}\|z\|_{L^{\infty}\left(0, \tau ; W^{1, \infty}(\Omega)\right)}+\|f\|_{L^{q}\left(0, \tau ; L^{\infty}(\Omega)\right)} ;
$$

where $M_{c, b}^{q} \max \left\{\|b\|_{L^{q}\left(0, \tau ; L^{\infty}(\Omega)\right)},\|c\|_{L^{q}\left(0, \tau ; L^{\infty}(\Omega)\right)}\right\}$;

(ii) if, moreover, $f$ and $z_{0}$ are non-negative, also $z$ is non-negative;

(iii) if, further, $f=0$ and $\inf _{x \in \Omega} z_{0}>0$, then $\left.\forall t \in\right] 0, \tau[$

$$
\inf _{x \in \Omega} z(t) \geq e^{-\int_{0}^{t}\left\|c\left(t^{\prime}\right)\right\|_{\infty, \Omega} d t^{\prime}} \inf _{x \in \Omega} z_{0} .
$$

Proof. (i) it follows easily from part (b) of Lemma 4.4, from (i-c) of Lemma 4.1 and, by Lemma 4.3, from estimates (4.4) and (4.11).

(ii) it follows from Lemmas 4.2 and 4.1 .

(iii) since there exists a $\left.\tau^{\prime} \in\right] 0, \tau\left[\right.$ such that $\zeta=1 / z \in C(] 0, \tau^{\prime}\left[; L^{\infty}(\Omega)\right)$, and since $z$ possesses partial derivatives a.e. in $] 0, \tau[\times \Omega$, by (ii-a) of Lemma $4.1 \zeta$ satisfies a.e. in $] 0, \tau^{\prime}[\times \Omega$ the equation

$$
\frac{\partial \zeta}{\partial t}+b \cdot \nabla \zeta-c \zeta=0
$$

the result readily follows from application to $\zeta$ of estimate (4.4).

Remark 4.7. Corollary 4.6 holds in particular in case $\left.\Omega=\Omega^{\prime} \times\right] m_{1}, m_{2}[$, where $\Omega^{\prime}$ is a bounded open set of $\mathbf{R}^{d-1}$ endowed with a strongly Lipschitz continuous boundary. In this case $b=\left(b^{\prime}, b_{m}\right)$, where $b^{\prime}$ takes values in $\mathbf{R}^{d-1}$ whereas $b_{m}$ takes values in $\mathbf{R},\left.n^{\prime} \cdot b^{\prime}\right|_{\partial \Omega^{\prime}}=0$, where $n^{\prime}$ is the exterior normal unit vector to $\partial \Omega^{\prime}$, and $b_{m}\left(t, x^{\prime}, m\right)=0$ for almost every $\left.\left(t, x^{\prime}\right) \in\right] 0, \tau\left[\times \Omega^{\prime}\right.$ if $m \in\left\{m_{1}, m_{2}\right\}$. The following corollary is a variant of this result.

Corollary 4.8. Let $\Omega$ be an open and bounded subset of $\mathbf{R}^{d}$ endowed with a strongly Lipschitz continuous boundary $\partial \Omega$, and let $\left.\Omega^{+}=\Omega \times\right] 0,+\infty[$, $\left.S_{\tau}=\right] 0, \tau\left[\times \Omega^{+}\right.$. Let, for some $q \in[1, \infty]$, be

(4.16) $b \in L^{q}(] 0, \tau\left[; W^{1, \infty}\left(\Omega^{+}\right)\right)^{d+1} c, f \in L^{q}(] 0, \tau\left[; W^{1, \infty}\left(\Omega^{+}\right)\right)$,

(4.17) $z_{0} \in W^{1, \infty}\left(\Omega^{+}\right)$,

(4.18) $b=\left(b_{x}, b_{m}\right)$, where $b_{x}$ takes values in $\mathbf{R}^{d}, b_{m}$ takes values in $\mathbf{R}$, 


$$
\left.\left.n \cdot b_{x}\right|_{\partial \Omega}=0, \quad \operatorname{supp} b_{m}(t, x, \cdot) \subseteq\left[m_{0}, M_{0}\right], \text { for a.e. }(t, x) \text { in }\right] 0, \tau[\times \Omega,
$$

for some positive $m_{0}$ and $M_{0}>m_{0}$.

Let be given the following problem:

$$
\begin{cases}\frac{\partial z}{\partial t}+b \cdot \nabla z+c z=f & \text { in } S_{\tau} \\ z(0, \cdot)=z_{0} & \text { in } \Omega^{+}\end{cases}
$$

Then

(a) there exists a unique solution $z$ lying in $L^{\infty}(] 0, \tau\left[; W^{1, \infty}\left(\Omega^{+}\right)\right) \cap$ $\cap W^{1, q}(] 0, \tau\left[; L^{\infty}\left(\Omega^{+}\right)\right)$of problem $(4.20)$; estimates (4.4) and (4.14) hold with $\Omega$ replaced by $\Omega^{+}$; in the space $L^{\infty}\left(S_{\tau}\right)$, the solution $z$ depends continuously on the data $z_{0}, b, c, f$; if, also, $f$ and $z_{0}$ are non-negative, then $z$ is non-negative too;

(b) furthermore, if we assume that

(4.21) $\operatorname{supp} z_{0}(x, \cdot) \subseteq\left[m_{0}, M_{0}\right] \quad \forall x \in \Omega$, and

(4.22) $\operatorname{supp} f(t, x \cdot) \subseteq\left[m_{0}, M_{0}\right]$, for a. e. $(t, x)$ in $[0, \tau] \times \Omega$, then

(4.23) $\operatorname{supp} z(t, x, \cdot) \subseteq\left[m_{0}, M_{0}\right]$ for a.e. $(t, x)$ in $[0, \tau] \times \Omega$.

Proof. (a): consider (4.20) in $] 0 . \tau[\times \Omega \times] m_{1}, m_{2}\left[\right.$, with $m_{1} \in\left[0, m_{0}\right], m_{2} \geq$ $M_{0}$. Remark 4.7 yields wellposedness in $L^{\infty}(] 0 . \tau\left[; W^{1, \infty}(\Omega \times] m_{1}, m_{2}[)\right) \cap$ $\cap W^{1, q}(] 0 . \tau\left[; L^{\infty}(\Omega \times] m_{1}, m_{2}[)\right)$. By the arbitrarity of $m_{1}$ and $m_{2}$, and by (4.14), (4.15) and application of (4.5), the result easily follows.

(b): for $m \notin] m_{0}, M_{0}[(4.20)$ yields

$$
\begin{cases}\frac{\partial z}{\partial t}+b_{x} \cdot \nabla_{x} z+c z=0 & \text { in } Q_{\tau} \\ z(0, \cdot)=0 & \text { in } \Omega\end{cases}
$$

hence $z=0$.

\section{The linearized system}

In order to prove Theorem 3.1 we preliminarily study a suitable linearization of system (Q). To this end we introduce:

(5.1) $\mathcal{S}_{0}(\pi, \sigma, \nu)=\nabla_{x} \cdot u+s_{l}^{\prime}\left(\pi-\pi_{l}\right)-s_{l} \frac{\partial \pi_{l}}{\partial m}-$ $-\frac{s_{l}}{m}\left(\pi-\pi_{l}\right)+K_{l s}+g_{l}\left[\pi-\pi_{l}\right]^{-}-J_{l}(\sigma)-J_{l s}(\nu)$, $\mathcal{S}_{+}(\pi, \sigma, \nu)=K_{s l} \nu+g_{a}\left[N^{*}-N(\sigma, \nu)\right]^{+}\left[\pi-\pi_{l}\right]^{+}+Q_{l}(\sigma, \sigma) ;$

(5.3) $\mathcal{N}_{0}(\pi, \sigma, \nu)=\nabla_{x} \cdot w+s_{s}^{\prime}\left(\pi-\pi_{s}\right)-$ $-\frac{s_{s}}{m}\left(\pi-\pi_{s}\right)+K_{s l}+g_{s}\left[\pi-\pi_{s}\right]^{-}-J_{s}(\nu)-J_{l s}(\sigma)$,

$\mathcal{N}_{+}(\sigma, \nu)=K_{l s} \sigma+Q_{s}(\nu, \nu)+Q_{l s}(\nu, \sigma)$. 
Our linearization of system $(\mathrm{Q})$ is:

$$
\left\{\begin{array}{l}
\frac{\partial \rho}{\partial t}+\nabla_{x} \cdot(\rho v)=0 \\
\frac{\partial \pi}{\partial t}+\nabla_{x} \cdot(\pi v)=\mathcal{P}(\pi, \bar{\sigma}, \bar{\nu}) \\
\frac{\partial \sigma}{\partial t}+u \cdot \nabla_{x} \sigma+s_{l}\left(\bar{\pi}-\pi_{l}\right) \frac{\partial \sigma}{\partial m}+\mathcal{S}_{0}(\bar{\pi}, \bar{\sigma}, \bar{\nu}) \sigma=\mathcal{S}_{+}(\bar{\pi}, \bar{\sigma}, \bar{\nu}) \\
\frac{\partial \nu}{\partial t}+w \cdot \nabla_{x} \nu+s_{s}\left(\bar{\pi}-\pi_{s}\right) \frac{\partial \nu}{\partial m}+\mathcal{N}_{0}(\bar{\pi}, \bar{\sigma}, \bar{\nu}) \nu=\mathcal{N}_{+}(\bar{\sigma}, \bar{\nu})
\end{array}\right.
$$

where $\bar{\pi}, \bar{\sigma}$ and $\bar{\nu}$ are given functions. We have:

Lemma 5.1. Assume that the temperature and velocities satisfy (3.1)-(3.5). If $\bar{\pi} \in L^{\infty}(] 0, \tau\left[; W^{1, \infty}(\Omega)\right)$ and $\bar{\sigma}, \bar{\nu} \in L^{\infty}(] 0, \tau\left[; W^{1, \infty}\left(\Omega^{+}\right)\right)$, then there exists one solution $(\rho, \pi, \sigma, \nu)$ of system $(L)$ satisfying initial conditions as in (3.6)(3.9) and such that

$$
\begin{array}{r}
\rho, \pi \in L^{\infty}(] 0, \tau\left[; W^{1, \infty}(\Omega)\right) \cap W^{1, q}(] 0, \tau\left[; L^{\infty}(\Omega)\right), \\
\sigma, \nu \in L^{\infty}(] 0, \tau\left[; W^{1, \infty}\left(\Omega^{+}\right)\right) \cap W^{1, q}(] 0, \tau\left[; L^{\infty}\left(\Omega^{+}\right)\right) ;
\end{array}
$$

moreover, if $\bar{\pi}, \bar{\sigma}$ and $\bar{\nu}$ are non negative, then also $\rho, \pi, \sigma$ and $\nu$ are non negative, and $\rho$ satisfies estimate (3.14).

Further, there exists a constant $C_{0}>0$, independent of $T, v, u, w$, such that the following estimates hold ( $q^{\prime}$ being the conjugate of $q$ ):

$$
\begin{aligned}
& \|\rho\|_{L^{\infty}\left(0, \tau ; W^{1, \infty}(\Omega)\right)} \leq \\
& \leq e^{\tau^{1 / q^{\prime}}\left(\|D v\|_{L^{q}\left(0, \tau ; L^{\infty}(\Omega)\right)}+\|\nabla \cdot v\|_{L^{q}\left(0, \tau ; W^{1, \infty}(\Omega)\right)}\right)}\left\|\rho_{0}\right\|_{W^{1, \infty}(\Omega)},
\end{aligned}
$$

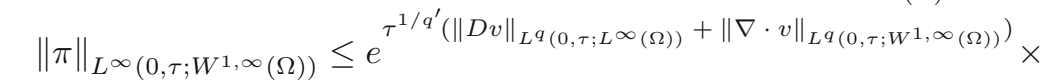

$$
\begin{aligned}
& \times e^{\tau C_{0}\left(\|\bar{\sigma}\|_{L^{\infty}\left(0, \tau ; W^{1, \infty}\left(\Omega^{+}\right)\right)}+\|\bar{\nu}\|_{L^{\infty}\left(0, \tau ; W^{1, \infty}\left(\Omega^{+}\right)\right)}\right)}\left[\left\|\pi_{0}\right\|_{W^{1, \infty}(\Omega)}+C_{0}(\tau+\right. \\
& \left.\left.+\tau^{1 / q^{\prime}}\|\nabla T\|_{L^{q}\left(0, \tau ; L^{\infty}(\Omega)\right)}\right)\left(\|\bar{\sigma}\|_{L^{\infty}\left(0, \tau ; W^{1, \infty}\left(\Omega^{+}\right)\right)}+\|\bar{\nu}\|_{L^{\infty}\left(0, \tau ; W^{1, \infty}\left(\Omega^{+}\right)\right)}\right)\right], \\
& \text {(5.7) }\|\sigma\|_{L^{\infty}\left(0, \tau ; W^{1, \infty}\left(\Omega^{+}\right)\right)} \leq
\end{aligned}
$$

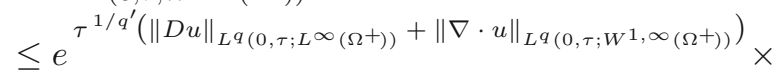

$$
\begin{aligned}
& \times e^{C_{0} \tau\left(\|\bar{\pi}\|_{L^{\infty}\left(0, \tau ; W^{1, \infty}(\Omega)\right)}+\|\bar{\sigma}\|_{L^{\infty}\left(0, \tau ; W^{1, \infty}\left(\Omega^{+}\right)\right)}+\|\bar{\nu}\|_{L^{\infty}\left(0, \tau ; W^{1, \infty}\left(\Omega^{+}\right)\right.}+1\right)} \times \\
& \times e^{C_{0} \tau^{1 / q^{\prime}}\|\nabla T\|_{L^{q}\left(0, \tau ; L^{\infty}(\Omega)\right)}}\left\{\left\|\sigma_{0}\right\|_{W^{1, \infty}\left(\Omega^{+}\right)}+C_{0}\left[\left(\tau^{1 / q^{\prime}}\|\nabla T\|_{L^{q}\left(0, \tau ; L^{\infty}(\Omega)\right)}+\right.\right.\right. \\
& \left.+\tau+\tau\|\bar{\pi}\|_{L^{\infty}\left(0, \tau ; W^{1, \infty}(\Omega)\right)}+\tau\|\bar{\sigma}\|_{L^{\infty}\left(0, \tau ; W^{1, \infty}\left(\Omega^{+}\right)\right)}\right) \times \\
& \left.\left.\times\left(1+\|\bar{\sigma}\|_{L^{\infty}\left(0, \tau ; W^{1, \infty}\left(\Omega^{+}\right)\right)}+\|\bar{\nu}\|_{L^{\infty}\left(0, \tau ; W^{1, \infty}\left(\Omega^{+}\right)\right)}\right)\right]\right\}, \\
& \text {(5.8) }\|\nu\|_{L^{\infty}\left(0, \tau ; W^{1, \infty}\left(\Omega^{+}\right)\right)} \leq \\
& \leq e^{\tau^{1 / q^{\prime}}\left(\|D w\|_{L^{q}\left(0, \tau ; L^{\infty}\left(\Omega^{+}\right)\right)}+\|\nabla \cdot w\|_{L^{q}\left(0, \tau ; W^{1, \infty}\left(\Omega^{+}\right)\right)}\right)} \times \\
& \times e^{C_{0} \tau\left(\|\bar{\pi}\|_{L^{\infty}\left(0, \tau ; W^{1, \infty}(\Omega)\right)}+\|\bar{\sigma}\|_{L^{\infty}\left(0, \tau ; W^{1, \infty}\left(\Omega^{+}\right)\right)}+\|\bar{\nu}\|_{L^{\infty}\left(0, \tau ; W^{1, \infty}\left(\Omega^{+}\right)\right.}+1\right)} \times \\
& \times e^{C_{0} \tau^{1 / q^{\prime}}\|\nabla T\|_{L^{q}\left(0, \tau ; L^{\infty}(\Omega)\right)}}\left[\left\|\nu_{0}\right\|_{W^{1, \infty}\left(\Omega^{+}\right)}+C_{0}\left[\left(\tau^{1 / q^{\prime}}\|\nabla T\|_{L^{q}\left(0, \tau ; L^{\infty}(\Omega)\right)}+\right.\right.\right. \\
& \left.\left.+\tau+\tau\|\bar{\nu}\|_{L^{\infty}\left(0, \tau ; W^{1, \infty}(\Omega)\right)}\right)\left(\|\bar{\sigma}\|_{L^{\infty}\left(0, \tau ; W^{1, \infty}\left(\Omega^{+}\right)\right)}+\|\bar{\nu}\|_{L^{\infty}\left(0, \tau ; W^{1, \infty}\left(\Omega^{+}\right)\right)}\right)\right] .
\end{aligned}
$$


Finally, if we have

$\operatorname{supp} \bar{\sigma}(t, x, \cdot), \operatorname{supp} \bar{\nu}(t, x, \cdot) \subseteq\left[m_{a}, M\right] \quad \forall(t, x) \in[0, \tau] \times \Omega$

then

(5.10) $\operatorname{supp} \sigma(t, x, \cdot), \operatorname{supp} \nu(t, x, \cdot) \subseteq\left[m_{a}, M\right] \quad \forall(t, x) \in[0, \tau] \times \Omega$.

Proof. The linearized system (L) is made up of indipendent equations. We verify at a stroke that the hypotheses of Corollaries 4.6 (for $\rho$ and for $\pi$ ) or 4.8 (for $\sigma$ and for $\nu$ ) are satisfied and that estimates (5.5)-(5.8) hold.

Consider first equation in $\rho$ with the initial condition $\rho_{0}$. In order to apply Corollary 4.6 we take

$$
b=v, \quad c=\nabla_{x} \cdot v, \quad f=0, \quad z_{0}=\rho_{0}, \quad z=\rho ;
$$

by (4.14) and Hölder's inequality we obtain (5.5). Application of (ii) and (iii) of Corollary 4.6 yields estimate (3.14).

Consider now the equation in $\pi$ with the initial condition $\pi_{0}$. In order to apply Corollary 4.6 we take

$$
\begin{aligned}
& b=v, c=\nabla_{x} \cdot v+F_{l}(\bar{\sigma})+F_{s}(\bar{\nu}), \quad f=F_{l}\left(\pi_{l} \bar{\sigma}\right)+\pi_{s} F_{s}(\bar{\nu}), \\
& z_{0}=\pi_{0}, \quad z=\pi
\end{aligned}
$$

we have, by (2.4) and (2.5), that for some constant $C_{F}$

$$
\begin{aligned}
& \left\|F_{l}(\bar{\sigma})\right\|_{W^{1, \infty}(\Omega)} \leq C_{F}\|\bar{\sigma}\|_{W^{1, \infty}\left(\Omega^{+}\right)}, \quad\left\|F_{s}(\bar{\nu})\right\|_{W^{1, \infty}(\Omega)} \leq C_{F}\|\bar{\nu}\|_{W^{1, \infty}\left(\Omega^{+}\right)}, \\
& \left\|F_{l}\left(\pi_{l}(T) \bar{\sigma}\right)\right\|_{W^{1, \infty}(\Omega)} \leq C_{F} \sup _{m}\left\|\pi_{l}(T)\right\|_{W^{1, \infty}(\Omega)}\|\bar{\sigma}\|_{W^{1, \infty}\left(\Omega^{+}\right)}
\end{aligned}
$$

by (2.3) and (2.23) it follows then that

$$
\begin{aligned}
\|f\|_{W^{1, \infty}(\Omega)} \leq & C_{F}\left[\left(\left\|\pi_{l}\right\|_{\infty}+\left\|\partial_{T} \pi_{l}\right\|_{\infty}\|\nabla T\|_{\infty}\right)\|\bar{\sigma}\|_{W^{1, \infty}\left(\Omega^{+}\right)}+\right. \\
& \left.+\left(\left\|\pi_{s}\right\|_{\infty}+\left\|\partial_{T} \pi_{s}\right\|_{\infty}\|\nabla T\|_{\infty}\right)\|\bar{\nu}\|_{W^{1, \infty}\left(\Omega^{+}\right)}\right]
\end{aligned}
$$

hence, by Hölder's inequality, for some constant $C_{0}$

$$
\begin{aligned}
& \tau^{1 / q^{\prime}}\|f\|_{L^{q}\left(0, \tau ; W^{1, \infty}(\Omega)\right)} \leq C_{0}\left(\tau+\tau^{1 / q^{\prime}}\|\nabla T\|_{L^{q}\left(0, \tau ; L^{\infty}(\Omega)\right)}\right) \times \\
& \quad \times\left(\|\bar{\sigma}\|_{L^{\infty}\left(0, \tau ; W^{1, \infty}\left(\Omega^{+}\right)\right)}+\|\bar{\nu}\|_{L^{\infty}\left(0, \tau ; W^{1, \infty}\left(\Omega^{+}\right)\right)}\right) ;
\end{aligned}
$$

by (4.14) estimate (5.6) readily follows; (ii) of Corollary 4.6 also applies.

Consider now the problem for the equation in $\sigma$ with initial condition $\sigma_{0}$. In order to apply Corollary 4.8, we assume

$$
b_{x}=u, b_{m}=s_{l}\left(\bar{\pi}-\pi_{l}\right), c=\mathcal{S}_{0}(\bar{\pi}, \bar{\sigma}, \bar{\nu}),
$$

(5.11) $f=\mathcal{S}_{+}(\bar{\pi}, \bar{\sigma}, \bar{\nu})$,

$$
z_{0}=\sigma_{0}, \quad z=\sigma
$$

by (2.6) $-(2.14)$ and by (2.23)-(2.25) we have then, for some $C>0$,

$$
\begin{aligned}
& \|D b\|_{\infty} \leq\|D u\|_{\infty}+\left\|s_{l}^{\prime}\right\|_{\infty}\left(\|\bar{\pi}\|_{\infty}+\left\|\pi_{l}\right\|_{\infty}\right)+ \\
& \quad+\left\|s_{l}\right\|_{\infty}\left(\|\nabla \bar{\pi}\|_{\infty}+\left\|\partial_{m} \pi_{l}\right\|_{\infty}+\left\|\partial_{T} \pi_{l}\right\|_{\infty}\|\nabla T\|_{\infty}\right) \leq \\
& \leq\|D u\|_{\infty}+C\left(\|\bar{\pi}\|_{W^{1, \infty}(\Omega)}+1+\|\nabla T\|_{L^{\infty}(\Omega)}\right),
\end{aligned}
$$


and also, by (5.1),

$$
\begin{aligned}
& \|c\|_{W^{1, \infty}\left(\Omega^{+}\right)} \leq\left\|\nabla_{x} \cdot u\right\|_{W^{1, \infty}\left(\Omega^{+}\right)}+\left(\left\|s_{l}^{\prime}\right\|_{\infty}+\left\|s_{l}^{\prime \prime}\right\|_{\infty}+\left\|\frac{s_{l}}{m}\right\|_{\infty}+\left\|\frac{s_{l}^{\prime}}{m}\right\|_{\infty}+\right. \\
& \left.\quad+\left\|\frac{s_{l}}{m^{2}}\right\|_{\infty}+\left\|g_{l}\right\|_{\infty}\right)\left(\|\bar{\pi}\|_{W^{1, \infty}\left(\Omega^{+}\right)}+\left\|\pi_{l}\right\|_{\infty}+\left\|\partial_{m} \pi_{l}\right\|_{\infty}+\right. \\
& \left.\quad+\left\|\partial_{T} \pi_{l}\right\|_{\infty}\|\nabla T\|_{L^{\infty}(\Omega)}\right)+\left(\left\|s_{l}^{\prime}\right\|_{\infty}+\left\|s_{l}^{\prime}\right\|_{\infty}\right)\left(\left\|\partial_{m} \pi_{l}\right\|_{\infty}+\left\|\partial_{m}^{2} \pi_{l}\right\|_{\infty}+\right. \\
& \left.\quad+\left\|\partial_{m, T}^{2} \pi_{l}\right\|_{\infty}\|\nabla T\|_{L^{\infty}(\Omega)}\right)+\left\|K_{l s}\right\|_{\infty}+\left\|\partial_{m} K_{l s}\right\|_{\infty}+ \\
& \quad+\left\|\partial_{T} K_{l s}\right\|_{\infty}\|\nabla T\|_{L^{\infty}(\Omega)}+M^{2}\left(\left\|\beta_{l}\right\|_{\infty}+\left\|\partial_{m} \beta_{l}\right\|_{\infty}\right)\|\bar{\sigma}\|_{W^{1, \infty}\left(\Omega^{+}\right)}+ \\
& \quad+M^{2}\left(\left\|Z_{l s}\right\|_{\infty}+\left\|\partial_{m} Z_{l} s\right\|_{\infty}\right)\|\bar{\nu}\|_{W^{1, \infty}\left(\Omega^{+}\right)} \leq \\
& \leq\left\|\nabla_{x} \cdot u\right\|_{W^{1, \infty}\left(\Omega^{+}\right)}+C\left(1+\|\bar{\pi}\|_{W^{1, \infty}\left(\Omega^{+}\right)}+\|\nabla T\|_{L^{\infty}(\Omega)}+\right. \\
& \left.\quad+\|\bar{\sigma}\|_{W^{1, \infty}\left(\Omega^{+}\right)}+\|\bar{\nu}\|_{W^{1, \infty}\left(\Omega^{+}\right)}\right)
\end{aligned}
$$

moreover, by (5.2),

$$
\begin{aligned}
&\|f\|_{W^{1, \infty}\left(\Omega^{+}\right)} \leq \\
& \leq\left(\left\|K_{s l}\right\|_{\infty}+\left\|\partial_{m} K_{s l}\right\|_{\infty}+\left\|\partial_{T} K_{s l}\right\|_{\infty}\|\nabla T\|_{L^{\infty}(\Omega)}\right)\|\bar{\nu}\|_{W^{1, \infty}\left(\Omega^{+}\right)}+ \\
&+\left(\left\|g_{a}\right\|_{\infty}+\left\|g_{a}^{\prime}\right\|_{\infty}\right)\left(\left\|N^{*}\right\|_{W^{1, \infty}(\Omega)}+C\|\bar{\sigma}\|_{W^{1, \infty}\left(\Omega^{+}\right)}+C\|\bar{\nu}\|_{W^{1, \infty}\left(\Omega^{+}\right)}\right) \times \\
& \times\left(\|\bar{\pi}\|_{W^{1, \infty}\left(\Omega^{+}\right)}+\left\|\pi_{l}\right\|_{\infty}+\left\|\partial_{m} \pi_{l}\right\|_{\infty}+\left\|\partial_{T^{\prime}} \pi_{l}\right\|_{\infty}\|\nabla T\|_{L^{\infty}(\Omega)}\right)+ \\
&+M\left(\left\|\beta_{l}\right\|_{\infty}+2\left\|\partial_{m} \beta_{l}\right\|_{\infty}\right)\|\bar{\sigma}\|_{W^{1, \infty}\left(\Omega^{+}\right)} \| \bar{\sigma}_{L^{\infty}\left(\Omega^{+}\right)} \leq \\
& \leq C\left(1+\|\bar{\pi}\|_{W^{1, \infty}(\Omega)}+\|\bar{\sigma}\|_{W^{1, \infty}\left(\Omega^{+}\right)}+\|\nabla T\|_{L^{\infty}(\Omega)}\right)(1+ \\
&\left.+\|\bar{\sigma}\|_{W^{1, \infty}\left(\Omega^{+}\right)}+\|\bar{\nu}\|_{W^{1, \infty}\left(\Omega^{+}\right)}\right)
\end{aligned}
$$

therefore, making use of Hölder's inequality, by (4.14) estimate (5.7) immediately follows; (ii) of Corollary 4.6 also applies.

Now, one can similarly proceed for the equation in $\nu$ : in this case we assume

$$
\begin{aligned}
& b_{x}=w, b_{m}=s_{s}\left(\bar{\pi}-\pi_{s}\right), c=\mathcal{N}_{0}(\bar{\pi}, \bar{\sigma}, \bar{\nu}), f=\mathcal{N}_{+}(\bar{\sigma}, \bar{\nu}), \\
& z_{0}=\nu_{0}, \quad z=\nu
\end{aligned}
$$

recalling (2.14)-(2.23) and (2.25), one easily finds, for some $C>0$,

$$
\|D b\|_{\infty} \leq\|D w\|_{\infty}+C\left(\|\bar{\pi}\|_{W^{1, \infty}(\Omega)}+1+\|\nabla T\|_{L^{\infty}(\Omega)}\right)
$$

and also, by (5.3),

$$
\begin{aligned}
& \|c\|_{W^{1, \infty}\left(\Omega^{+}\right)} \leq\left\|\nabla_{x} \cdot w\right\|_{W^{1, \infty}\left(\Omega^{+}\right)}+C\left(1+\|\bar{\pi}\|_{W^{1, \infty}\left(\Omega^{+}\right)}+\|\nabla T\|_{L^{\infty}(\Omega)}+\right. \\
& \left.\quad+\|\bar{\sigma}\|_{W^{1, \infty}\left(\Omega^{+}\right)}+\|\bar{\nu}\|_{W^{1, \infty}\left(\Omega^{+}\right)}\right)
\end{aligned}
$$


moreover, by (5.4),

$$
\begin{aligned}
&\|f\|_{W^{1, \infty}\left(\Omega^{+}\right)} \leq \\
& \leq\left(\left\|K_{l s}\right\|_{\infty}+\left\|\partial_{m} K_{l s}\right\|_{\infty}+\left\|\partial_{T} K_{l s}\right\|_{\infty}\|\nabla T\|_{L^{\infty}(\Omega)}\right)\|\bar{\sigma}\|_{W^{1, \infty}\left(\Omega^{+}\right)}+ \\
&+M\left(\left\|\beta_{s}\right\|_{\infty}+2\left\|\partial_{m} \beta_{s}\right\|_{\infty}\right)\|\bar{\nu}\|_{W^{1, \infty}\left(\Omega^{+}\right)}\|\bar{\nu}\|_{L^{\infty}\left(\Omega^{+}\right)}+C\left(\left\|Z_{l s}\right\|_{\infty}+\right. \\
&\left.+\left\|\partial_{m} Z_{l s}\right\|_{\infty}+\left\|\partial_{m^{\prime}} Z_{l s}\right\|_{\infty}\right)\|\bar{\sigma}\|_{W^{1, \infty}\left(\Omega^{+}\right)}\|\bar{\nu}\|_{W^{1, \infty}\left(\Omega^{+}\right)} \leq \\
& \leq C\left(1+\|\nabla T\|_{L^{\infty}(\Omega)}+\|\bar{\nu}\|_{W^{1, \infty}\left(\Omega^{+}\right)}\right)\left(\|\bar{\sigma}\|_{W^{1, \infty}\left(\Omega^{+}\right)}+\|\bar{\nu}\|_{W^{1, \infty}\left(\Omega^{+}\right)}\right)
\end{aligned}
$$

estimate (5.8) readily follows; (ii) of Corollary 4.6 also applies.

Let us now prove the last assertion of the lemma: if we denote by $f$, as in (5.11), the right-hand side of the equation in $\sigma$, it is easily checked, by (5.9), by (2.6)-(2.14) and by (2.24)-(2.25), that

$$
\operatorname{supp} f(t, x, \cdot) \subseteq\left[m_{a}, M\right] \text { a.e. in }[0, \tau] \times \Omega \text {; }
$$

therefore, in order to obtain the first assertion of (5.10), it is sufficient to apply the last part of Corollary 4.8. By (2.14)-(2.23) and (2.25) one can similarly proceed for $\nu$.

Corollary 5.2. There exists $\left.\left.\tau_{1} \in\right] 0, \tau\right]$, depending continuously on $\left\|\pi_{0}\right\|_{W^{1, \infty}(\Omega)}$, $\left\|\sigma_{0}\right\|_{W^{1, \infty}\left(\Omega^{+}\right)}$and $\left\|\nu_{0}\right\|_{W^{1, \infty}\left(\Omega^{+}\right)}$, such that if

$$
\bar{\pi} \in L^{\infty}(] 0, \tau_{1}\left[; W^{1, \infty}(\Omega)\right), \bar{\sigma}, \bar{\nu} \in L^{\infty}(] 0, \tau_{1}\left[; W^{1, \infty}\left(\Omega^{+}\right)\right)
$$

are non negative and satisfy the hypotheses of Lemma 5.1, with

$$
\begin{aligned}
& \|\bar{\pi}\|_{L^{\infty}(] 0, \tau_{1}\left[; W^{1, \infty}(\Omega)\right)} \leq 2\left\|\pi_{0}\right\|_{W^{1, \infty}(\Omega)}+1, \\
& \|\bar{\sigma}\|_{L^{\infty}(] 0, \tau_{1}\left[; W^{1, \infty}\left(\Omega^{+}\right)\right)} \leq 2\left\|\sigma_{0}\right\|_{W^{1, \infty}\left(\Omega^{+}\right)}+1, \\
& \|\bar{\nu}\|_{L^{\infty}(] 0, \tau_{1}\left[; W^{1, \infty}\left(\Omega^{+}\right)\right)} \leq 2\left\|\nu_{0}\right\|_{W^{1, \infty}\left(\Omega^{+}\right)}+1,
\end{aligned}
$$

then the solution $(\rho, \pi, \sigma, \nu)$ (with non negative components) of system $(L)$, with initial conditions as in (3.6)-(3.9), satisfies the conditions

$$
\begin{aligned}
& \|\pi\|_{L^{\infty}(] 0, \tau_{1}\left[; W^{1, \infty}(\Omega)\right)} \leq 2\left\|\pi_{0}\right\|_{W^{1, \infty}(\Omega)}+1, \\
& \|\sigma\|_{L^{\infty}(] 0, \tau_{1}\left[; W^{1, \infty}\left(\Omega^{+}\right)\right)} \leq 2\left\|\sigma_{0}\right\|_{W^{1, \infty}\left(\Omega^{+}\right)}+1, \\
& \|\nu\|_{L^{\infty}(] 0, \tau_{1}\left[; W^{1, \infty}\left(\Omega^{+}\right)\right)} \leq 2\left\|\nu_{0}\right\|_{W^{1, \infty}\left(\Omega^{+}\right)}+1, \\
& \operatorname{supp} \sigma(t, x, \cdot), \operatorname{supp} \nu(t, x, \cdot) \subseteq\left[m_{a}, M\right] \quad \forall(t, x) \in\left[0, \tau_{1}\right] \times \Omega .
\end{aligned}
$$

Proof. It follows immediately from Lemma 5.1.

\section{Proof of Theorem 3.1}

\subsection{Existence and uniqueness}

The equation in $\rho$ being linear, existence, uniqueness and estimate (3.14) for $\rho$ result from Lemma 5.1. It suffices now to consider the equations in $\pi, \sigma$ and $\nu$. Let $\left.t \in] 0, \tau_{1}\right]$, where $\tau_{1}$ is as in Corollary 5.2. Let us define in $L^{\infty}\left(Q_{t}\right) \times L^{\infty}\left(S_{t}\right) \times L^{\infty}\left(S_{t}\right)$ the closed set $\mathcal{C}_{t}$ of all $(\pi, \sigma, \nu)$ such that 


$$
\left\{\begin{array}{l}
\pi \in L^{\infty}(] 0, t\left[; W^{1, \infty}(\Omega)\right) ; \quad \sigma, \nu \in L^{\infty}(] 0, t\left[; W^{1, \infty}\left(\Omega^{+}\right)\right) ; \\
\pi, \sigma, \nu \geq 0, \\
\operatorname{supp} \sigma\left(t^{\prime}, x, \cdot\right), \operatorname{supp} \nu\left(t^{\prime}, x, \cdot\right) \subseteq\left[m_{a}, M\right] \quad \forall\left(t^{\prime}, x\right) \in[0, t] \times \Omega \\
\|\pi\|_{L^{\infty}\left(0, t ; W^{1, \infty}(\Omega)\right)} \leq 2\left\|\pi_{0}\right\|_{W^{1, \infty}(\Omega)}+1, \\
\|\sigma\|_{L^{\infty}\left(0, t ; W^{1, \infty}\left(\Omega^{+}\right)\right)} \leq 2\left\|\sigma_{0}\right\|_{W^{1, \infty}\left(\Omega^{+}\right)}+1 \\
\|\nu\|_{L^{\infty}\left(0, t ; W^{1, \infty}\left(\Omega^{+}\right)\right)} \leq 2\left\|\nu_{0}\right\|_{W^{1, \infty}\left(\Omega^{+}\right)}+1
\end{array}\right.
$$

Consider, for any $t$ in $\left.] 0, \tau_{1}\right]$, the operator $G_{t}: \mathcal{C}_{t} \rightarrow L^{\infty}\left(Q_{t}\right) \times L^{\infty}\left(S_{t}\right) \times$ $L^{\infty}\left(S_{t}\right)$ such that $G_{t}(\bar{\pi}, \bar{\sigma}, \bar{\nu})=(\pi, \sigma, \nu)$, where $(\rho, \pi, \sigma, \nu)$ is the solution of system (L) with initial conditions as in (3.6)-(3.9).

By virtue of Corollary 5.2, the operator $G_{t}$ maps $\mathcal{C}_{t}$ into itself. We are going to prove that there exists a $\left.\left.\tau^{*} \in\right] 0, \tau_{1}\right]$ such that $G_{\tau^{*}}$ is a contraction. In order to show this, we consider, given $\left(\bar{\pi}_{i}, \bar{\sigma}_{i}, \bar{\nu}_{i}\right) \in \mathcal{C}_{t}$ with $i \in\{1,2\}$, $G_{t}\left(\bar{\pi}_{i}, \bar{\sigma}_{i}, \bar{\nu}_{i}\right)=\left(\pi_{i}, \sigma_{i}, \nu_{i}\right)$, and define

$$
\begin{array}{ll}
\bar{\Pi}=\bar{\pi}_{2}-\bar{\pi}_{1}, \quad \bar{\Sigma}=\bar{\sigma}_{2}-\bar{\sigma}_{1}, \quad \overline{\mathrm{N}}=\bar{\nu}_{2}-\bar{\nu}_{1}, \\
\Pi=\pi_{2}-\pi_{1}, \quad \Sigma=\sigma_{2}-\sigma_{1}, \quad \mathrm{~N}=\nu_{2}-\nu_{1} .
\end{array}
$$

Applying the perturbation estimate (4.5) to $z=\pi_{2}$ and $z^{\prime}=\pi_{1}$, with $b=b^{\prime}=v, c=\nabla \cdot v+F_{l}\left(\bar{\sigma}_{2}\right)+F_{s}\left(\bar{\nu}_{2}\right), c^{\prime}=\nabla \cdot v+F_{l}\left(\bar{\sigma}_{1}\right)+F_{s}\left(\bar{\nu}_{1}\right)$, $f=F_{l}\left(\pi_{l} \bar{\sigma}_{2}\right)+\pi_{s} F_{s}\left(\bar{\nu}_{2}\right), f^{\prime}=F_{l}\left(\pi_{l} \bar{\sigma}_{1}\right)+\pi_{s} F_{s}\left(\bar{\nu}_{1}\right)$, one finds, recalling $(3.2)$, (6.1), (2.1)-(2.5) and (2.23), that, for some constant $C$,

$$
\begin{aligned}
& \|\Pi\|_{\infty, Q_{t}} \leq t e^{t^{1 / q^{\prime}}\|\nabla \cdot v\|_{L^{q}\left(0, t ; L^{\infty}(\Omega)\right)}+2 C t\left(\left\|\sigma_{0}\right\|_{W^{1, \infty}\left(\Omega^{+}\right)}+\left\|\nu_{0}\right\|_{W^{1, \infty}\left(\Omega^{+}\right)}+2\right)} \times \\
& \times C\left(2\left\|\pi_{0}\right\|_{W^{1, \infty}(\Omega)}+1+\max \left\{\left\|\pi_{l}\right\|_{\infty},\left\|\pi_{s}\right\|_{\infty}\right\}\right)\left(\|\bar{\Sigma}\|_{\infty, Q_{t}}+\|\overline{\mathrm{N}}\|_{\infty, Q_{t}}\right) .
\end{aligned}
$$

Applying the perturbation estimate (4.5) to $z=\sigma_{2}$ and $z^{\prime}=\sigma_{1}$, with $b=\left(u, s_{l}\left[\bar{\pi}_{2}-\pi_{l}\right]\right), b^{\prime}=\left(u, s_{l}\left[\bar{\pi}_{1}-\pi_{l}\right]\right), c=\mathcal{S}_{0}\left(\bar{\pi}_{2}, \bar{\sigma}_{2}, \bar{\nu}_{2}\right), c^{\prime}=\mathcal{S}_{0}\left(\bar{\pi}_{1}, \bar{\sigma}_{1}, \bar{\nu}_{1}\right)$, $f=\mathcal{S}_{+}\left(\bar{\pi}_{2}, \bar{\sigma}_{2}, \bar{\nu}_{2}\right), f^{\prime}=\mathcal{S}_{+}\left(\bar{\pi}_{1}, \bar{\sigma}_{1}, \bar{\nu}_{1}\right)$, one also finds, by virtue of $(3.3),(5.1)$, (5.2), (6.1), (2.6)-(2.14) and (2.23)-(2.25),

$$
\begin{aligned}
& \|\Sigma\|_{\infty, S_{t}} \leq t e^{t^{1 / q^{\prime}}\left\|\nabla_{x} \cdot u\right\|_{L^{q}\left(0, t ; L^{\infty}\left(\Omega^{+}\right)\right)}} \times \\
& \times e^{t\left[\left(\left\|s_{l}^{\prime}\right\|_{\infty}+\left\|\frac{s_{l}}{m}\right\|_{\infty}+\left\|g_{l}\right\|_{\infty}\right)\left(2\left\|\pi_{0}\right\|_{W^{1, \infty}(\Omega)}+1+\left\|\pi_{l}\right\|_{\infty}\right)+\left\|s_{l}\right\|_{\infty}\left\|\partial_{m} \pi_{l}\right\|_{\infty}\right]} \times \\
& \times e^{t\left[\left\|K_{l s}\right\|_{\infty}+2 M^{2}\left(\left\|\beta_{l}\right\|_{\infty}+\left\|Z_{l s}\right\|_{\infty}\right)\left(\left\|\sigma_{0}\right\|_{W^{1, \infty}\left(\Omega^{+}\right)}+\left\|\nu_{0}\right\|_{W^{1, \infty}\left(\Omega^{+}\right)}+1\right)\right]} \times \\
& \times\left\{( 2 \| \sigma _ { 0 } \| _ { W ^ { 1 , \infty } ( \Omega ^ { + } ) } + 1 ) \left[\left(\left\|s_{l}\right\|_{\infty}+\left\|s_{l}^{\prime}\right\|_{\infty}+\left\|\frac{s_{l}}{m}\right\|_{\infty}+\left\|g_{l}\right\|_{\infty}\right)\|\bar{\Pi}\|_{\infty, Q_{t}}+\right.\right. \\
& \left.+M^{2}\left\|\beta_{l}\right\|_{\infty}\|\bar{\Sigma}\|_{\infty, S_{t}}+M^{2}\left\|Z_{l s}\right\|_{\infty}\|\overline{\mathrm{N}}\|_{\infty, S_{t}}\right]+\left\|K_{l s}\right\|_{\infty}\|\overline{\mathrm{N}}\|_{\infty, S_{t}}+ \\
& +\left\|g_{a}\right\|_{\infty}\left[C\left(\|\bar{\Sigma}\|_{\infty, S_{t}}+\|\overline{\mathrm{N}}\|_{\infty, S_{t}}\right)\left(2\left\|\pi_{0}\right\|_{W^{1, \infty}(\Omega)}+1+\left\|\pi_{l}\right\|_{\infty}\right)+\right. \\
& \left.+\left(\left\|N^{*}\right\|_{\infty}+2 C\left[\left\|\sigma_{0}\right\|_{W^{1, \infty}\left(\Omega^{+}\right)}+\left\|\nu_{0}\right\|_{W^{1, \infty}\left(\Omega^{+}\right)}+1\right]\right)\|\bar{\Pi}\|_{\infty, Q_{t}}\right]+ \\
& \left.+M^{2}\left\|\beta_{l}\right\|_{\infty}\left(2\left\|\sigma_{0}\right\|_{W^{1, \infty}\left(\Omega^{+}\right)}+1\right)\|\bar{\Sigma}\|_{\infty, S_{t}}\right\} .
\end{aligned}
$$


A similar estimate regards N. Thus, one finds that, for some positive constant $C_{1}$,

$$
\begin{aligned}
& \|\Pi\|_{L^{\infty}\left(Q_{t}\right)}+\|\Sigma\|_{L^{\infty}\left(S_{t}\right)}+\|\mathrm{N}\|_{L^{\infty}\left(S_{t}\right)} \leq \\
& \leq C_{1} t e^{C_{1}\left(t^{1 / q^{\prime}}+t\right)}\left(\|\bar{\Pi}\|_{L^{\infty}\left(Q_{t}\right)}+\|\bar{\Sigma}\|_{L^{\infty}\left(S_{t}\right)}+\|\overline{\mathrm{N}}\|_{L^{\infty}\left(S_{t}\right)}\right) .
\end{aligned}
$$

Hence, if $t=\tau^{*}$ is such that $0<C_{1} \tau^{*} e^{C_{1}\left[\left(\tau^{*}\right)^{1 / q^{\prime}}+\tau^{*}\right]}<1, G_{\tau^{*}}$ is a contraction. Let then $(\pi, \sigma, \nu)$ be the fixed point of $G_{\tau^{*}}$; by Lemma 5.1 it lies in the required space and $(\rho, \pi, \sigma, \nu)$ is the solution of problem $(\mathrm{Q})$. Note that a common $\tau^{*}$ may be chosen for initial data and divergences of velocities in a neighbourhood of $\left(\pi_{0}, \sigma_{0}, \nu_{0}, \nabla \cdot v, \nabla_{x} \cdot u, \nabla_{x} \cdot w\right)$ in $W^{1, \infty}(\Omega) \times W^{1, \infty}\left(\Omega^{+}\right)^{2} \times$ $L^{q}(] 0, \tau\left[; L^{\infty}(\Omega)\right) \times L^{q}(] 0, \tau\left[; L^{\infty}\left(\Omega^{+}\right)\right)^{2}$.

\subsection{Continuous dependence on data, velocities and temperature}

We assume that, for $i=1,2, T=T_{i}, v=v_{i}, u=u_{i}$ and $w=w_{i}$ satisfy respectively conditions (3.1), (3.2), (3.3) and (3.4), and that also condition (3.5) is satisfied. We indicate with $\left(\rho_{i}, \pi_{i}, \sigma_{i}, \nu_{i}\right)$ the corresponding solution of system (Q) which satisfies the initial condition (3.6)-(3.9) with $\left(\rho_{0}, \pi_{0}, \sigma_{0}, \nu_{0}\right)=\left(\rho_{0 i}, \pi_{0 i}, \sigma_{0 i}, \nu_{0 i}\right)$. As we have shown, one has, for any sufficiently small $t$,

$$
\begin{aligned}
& \rho_{i}, \pi_{i} \in L^{\infty}(] 0, t\left[; W^{1, \infty}(\Omega)\right) \cap W^{1, q}(] 0, t\left[; L^{\infty}(\Omega)\right), \\
& \sigma_{i}, \nu_{i} \in L^{\infty}(] 0, t\left[; W^{1, \infty}\left(\Omega^{+}\right)\right) \cap W^{1, q}(] 0, t\left[; L^{\infty}\left(\Omega^{+}\right)\right) .
\end{aligned}
$$

Set

$$
\begin{aligned}
& T=T_{2}-T_{1}, \quad V=v_{2}-v_{1}, \quad U=u_{2}-u_{1}, \quad W=w_{2}-w_{1}, \\
& \mathrm{P}=\rho_{2}-\rho_{1}, \quad \Pi=\pi_{2}-\pi_{1}, \quad \Sigma=\sigma_{2}-\sigma_{1}, \quad \mathrm{~N}=\nu_{2}-\nu_{1}
\end{aligned}
$$

taking the differences between the equations in $\rho_{1}, \pi_{1}, \sigma_{1}, \nu_{1}$ and in $\rho_{2}, \pi_{2}, \sigma_{2}$, $\nu_{2}$ we obtain

$$
\begin{aligned}
& \frac{\partial \mathrm{P}}{\partial t}=-\nabla_{x} \rho_{2} \cdot V-\rho_{2} \nabla_{x} \cdot V-v_{1} \cdot \nabla_{x} \mathrm{P}-\left(\nabla_{x} \cdot v_{1}\right) \mathrm{P} \\
& \quad \frac{\partial \Pi}{\partial t}=-\nabla_{x} \pi_{2} \cdot V-\pi_{2} \nabla_{x} \cdot V-v_{1} \cdot \nabla_{x} \Pi-\left(\nabla_{x} \cdot v_{1}\right) \Pi- \\
& -F_{l}\left(\Pi \sigma_{2}\right)-F_{s}\left(\nu_{2}\right) \Pi-F_{l}\left(\left[\pi_{l}\left(T_{2}\right)-\pi_{l}\left(T_{1}\right)\right] \sigma_{2}\right)-F_{s}\left(\nu_{2}\right)\left[\pi_{s}\left(T_{2}\right)-\pi_{s}\left(T_{1}\right)\right]- \\
& -F_{l}\left(\left[\pi_{1}-\pi_{l}\left(T_{1}\right)\right] \Sigma\right)-F_{s}(\mathrm{~N})\left[\pi_{1}-\pi_{s}\left(T_{1}\right)\right] \\
& \quad \frac{\partial \Sigma}{\partial t}=-\nabla_{x} \sigma_{2} \cdot U-\sigma_{2} \nabla_{x} \cdot U-\sigma_{2}\left[K_{l s}\left(T_{2}\right)-K_{l s}\left(T_{1}\right)\right]+ \\
& +\nu_{2}\left[K_{s l}\left(T_{2}\right)-K_{s l}\left(T_{1}\right)\right]+\left[\left(-s_{l}^{\prime}+\frac{s_{l}}{m}\right) \sigma_{2}-s_{l} \frac{\partial \sigma_{2}}{\partial m}\right] \times \\
& \times\left[\Pi-\left(\pi_{l}\left(T_{2}\right)-\pi_{l}\left(T_{1}\right)\right)\right]-s_{l}\left[\frac{\partial}{\partial m}\left(\pi_{l}\left(T_{2}\right)-\pi_{l}\left(T_{1}\right)\right)\right] \sigma_{2}-
\end{aligned}
$$




$$
\begin{aligned}
- & \sigma_{2} g_{l}\left[\left[\pi_{2}-\pi_{l}\left(T_{2}\right)\right]^{-}-\left[\pi_{1}-\pi_{l}\left(T_{1}\right)\right]^{-}\right]+ \\
+ & g_{a}\left[N^{*}-N\left(\sigma_{1}, \nu_{1}\right)\right]^{+}\left[\left[\pi_{2}-\pi_{l}\left(T_{2}\right)\right]^{+}-\left[\pi_{1}-\pi_{l}\left(T_{1}\right)\right]^{+}\right]+ \\
+ & {\left[-\nabla_{x} \cdot u_{1}+\left(-s_{l}^{\prime}+\frac{s_{l}}{m}\right)\left[\pi_{1}-\pi_{l}\left(T_{1}\right)\right]-s_{l} \frac{\partial \pi_{l}}{\partial m}\left(T_{1}\right)-K_{l s}\left(T_{1}\right)-\right.} \\
- & \left.g_{l}\left[\pi_{1}-\pi_{l}\left(T_{1}\right)\right]^{-}+J_{l}\left(\sigma_{1}\right)+J_{l s}\left(\nu_{1}\right)\right] \Sigma+Q_{l}\left(\Sigma, \sigma_{2}\right)+Q_{l}\left(\sigma_{1}, \Sigma\right)+ \\
+ & \sigma_{2} J_{l}(\Sigma)-u_{1} \cdot \nabla_{x} \Sigma-s_{l}\left[\pi_{1}-\pi_{l}\left(T_{1}\right)\right] \frac{\partial \Sigma}{\partial m}+K_{s l}\left(T_{1}\right) \mathrm{N}+\sigma_{2} J_{l s}(\mathrm{~N})+ \\
+ & g_{a}\left[\pi_{2}-\pi_{l}\left(T_{2}\right)\right]^{+}\left[\left[N^{*}-N\left(\sigma_{2}, \nu_{2}\right)\right]^{+}-\left[N^{*}-N\left(\sigma_{1}, \nu_{1}\right)\right]^{+}\right], \\
& \frac{\partial \mathrm{N}}{\partial t}=-\nabla_{x} \nu_{2} \cdot W-\nu_{2} \nabla_{x} \cdot W-\nu_{2}\left[K_{s l}\left(T_{2}\right)-K_{s l}\left(T_{1}\right)\right]+ \\
+ & \sigma_{2}\left[K_{l s}\left(T_{2}\right)-K_{l s}\left(T_{1}\right)\right]+\left[\left(-s_{s}^{\prime}+\frac{s_{s}}{m}\right) \nu_{2}-s_{s} \frac{\partial \nu_{2}}{\partial m}\right] \times \\
\times & {\left[\Pi-\left(\pi_{s}\left(T_{2}\right)-\pi_{s}\left(T_{1}\right)\right)\right]-\nu_{2} g_{s}\left[\left[\pi_{2}-\pi_{s}\left(T_{2}\right)\right]^{-}-\left[\pi_{1}-\pi_{s}\left(T_{1}\right)\right]\right.} \\
+ & {\left[-\nabla \cdot w_{1}+\left(-s_{s}^{\prime}+\frac{s_{s}}{m}\right)\left[\pi_{1}-\pi_{s}\left(T_{1}\right)\right]-K_{s l}\left(T_{1}\right)-\right.} \\
- & \left.g_{s}\left[\pi_{1}-\pi_{s}\left(T_{1}\right)\right]^{-}+J_{s}\left(\nu_{1}\right)+J_{l s}\left(\sigma_{1}\right)\right] \mathrm{N}+Q_{s}\left(\mathrm{~N}, \nu_{2}\right)+Q_{s}\left(\nu_{1}, \mathrm{~N}\right)+ \\
+ & Q_{l s}\left(\mathrm{~N}, \sigma_{1}\right)+\nu_{2} J_{s}(\mathrm{~N})-w_{1} \cdot \nabla \mathrm{N}-s_{s}\left[\pi_{1}-\pi_{s}\left(T_{1}\right)\right] \frac{\partial \mathrm{N}}{\partial m}+ \\
+ & K_{l s}\left(T_{1}\right) \Sigma+\nu_{2} J_{l s}(\Sigma)+Q_{l s}\left(\nu_{2}, \Sigma\right) .
\end{aligned}
$$

We may now apply estimate (4.4) to the equations (6.2)-(6.5) - with $\Omega$ replaced by $\Omega^{+}$if the case may be-by taking in each equation $c=0$, and respectively

$$
b=v_{1}, b=v_{1}, b=\left(u_{1}, s_{l}\left[\pi_{1}-\pi_{l}\left(T_{1}\right)\right]\right), \quad b=\left(w_{1}, s_{s}\left[\pi_{1}-\pi_{s}\left(T_{1}\right)\right]\right),
$$

whereas $f$ will encompass all other terms of the right-hand side. After summing, majorizing by virtue of (2.23) and (6.1), and applying Gronwall's lemma, one gets that, for some $C_{2}>0$,

$$
\begin{aligned}
& \|\mathrm{P}\|_{L^{\infty}\left(Q_{t}\right)}+\|\Pi\|_{L^{\infty}\left(Q_{t}\right)}+\|\Sigma\|_{L^{\infty}\left(S_{t}\right)}+\|\mathrm{N}\|_{L^{\infty}\left(S_{t}\right)} \leq \\
& \leq e^{C_{2} t}\left[\|\mathrm{P}(0)\|_{L^{\infty}(\Omega)}+\|\Pi(0)\|_{L^{\infty}(\Omega)}+\|\Sigma(0)\|_{L^{\infty}\left(\Omega^{+}\right)}+\|\mathrm{N}(0)\|_{L^{\infty}\left(\Omega^{+}\right)}+\right. \\
& \quad+C_{2} \int_{0}^{t}\left(\left\|T\left(t^{\prime}\right)\right\|_{L^{\infty}(\Omega)}+\left\|V\left(t^{\prime}\right)\right\|_{L^{\infty}(\Omega)}+\left\|\nabla \cdot V\left(t^{\prime}\right)\right\|_{L^{\infty}(\Omega)}+\right. \\
& \quad+\left\|U\left(t^{\prime}\right)\right\|_{L^{\infty}\left(\Omega^{+}\right)}+\left\|\nabla_{x} \cdot U\left(t^{\prime}\right)\right\|_{L^{\infty}\left(\Omega^{+}\right)}+ \\
& \left.\left.\quad+\left\|W\left(t^{\prime}\right)\right\|_{L^{\infty}\left(\Omega^{+}\right)}+\left\|\nabla_{x} \cdot W\left(t^{\prime}\right)\right\|_{L^{\infty}\left(\Omega^{+}\right)}\right) d t^{\prime}\right] \leq \\
& \leq e^{C_{2} t}\left[\|\mathrm{P}(0)\|_{L^{\infty}(\Omega)}+\|\Pi(0)\|_{L^{\infty}(\Omega)}+\|\Sigma(0)\|_{L^{\infty}\left(\Omega^{+}\right)}+\|\mathrm{N}(0)\|_{L^{\infty}\left(\Omega^{+}\right)}+\right. \\
& \quad C_{2} t^{1 / q^{\prime}}\left(\|T\|_{L^{q}\left(0, t ; L^{\infty}(\Omega)\right)}+\|V\|_{L^{q}\left(0, t ; L^{\infty}(\Omega)\right)}+\|\nabla \cdot V\|_{L^{q}\left(0, t ; L^{\infty}(\Omega)\right)}+\right. \\
& \quad+\|U\|_{L^{q}\left(0, t ; L^{\infty}\left(\Omega^{+}\right)\right)}+\left\|\nabla_{x} \cdot U\right\|_{L^{q}\left(0, t ; L^{\infty}\left(\Omega^{+}\right)\right)}+ \\
& \left.\left.\quad+\|W\|_{L^{q}\left(0, t ; L^{\infty}\left(\Omega^{+}\right)\right)}+\left\|\nabla_{x} \cdot W\right\|_{L^{q}\left(0, t ; L^{\infty}\left(\Omega^{+}\right)\right)}\right)\right],
\end{aligned}
$$

and this completes the proof. 


\section{Concluding remarks}

\subsection{Relationships among velocities}

We have supposed until now that the velocities $v, u, w$ are independent of each other. We are now going to briefly discuss which relationships may be assumed to exist among them and how Theorem 3.1 can be applied (we still assume to be a good approximation that dry air and vapor have the same velocity $v$ ).

Water droplets and ice particles are subject to the force of gravity and to friction with air. If $u-v$ (resp. $w-v$ ) is small, the latter may be assumed to be proportional to $\eta m^{1 / 3}(u-v)$ (resp. $\eta m^{1 / 3}(w-v)$ ), where $\eta$ is the air viscosity, which in turn depends on the temperature; for higher values of $|u-v|$, friction becomes proportional to $\rho m^{2 / 3}(u-v)^{2}$ (we neglect here friction with vapor). As a result, the velocities of droplets or ice particles asymptotically approach - if $\eta$ (or $\rho$ ) is constant - terminal velocities which depend only on their masses.

(a) As a first approximation one may then assume that $u(t, x, m)=$ $v(t, x)+\bar{u}(m, \eta(T(t, x)), \rho(t, x))$ and $w(t, x, m)=v(t, x)+\bar{w}(m, \eta(T(t, x)))$, where $\bar{u}$ and $\bar{w}$ are the terminal velocities relative to the mass $m$ (or functions thereof) when $\eta$ and $\rho$ take the constant values $\eta(T(t, x))$ and $\rho(t, x)$.

(b) For a better approximation, given $u(0, x, m)$ (e.g. through a suitable function of $\bar{u}(m, T(t, x), \rho(t, x))$ if $\sigma(0, x, m)=0)$, one can solve the Cauchy problem for the ordinary differential system in $u-v$

$$
m \frac{\partial}{\partial t}(u-v)(t, x, m)=\varphi_{l}(m,(u-v)(t, x, m), \eta(T(t, x)), \rho(t, x))-\nabla \Phi(x)
$$

where $\varphi_{l}$ is friction with air and $\Phi$ is the geopotential. Taking into account the Coriolis and the buoyant forces does not substantially change the form of this system. A similar one regards $w-v$.

In both cases one finds $u$ and $w$ as functions of $(t, x, m, \eta(T(t, x)), \rho(t, x))$ (in [19], as well as in [4], the dependence on $\rho$ is neglected, and $\eta$ is supposed to be constant), which we assume to be bounded with bounded derivatives with respect to $T$ and to $\rho$, up to the second order.

Taking then $v \in L^{q}(] 0, \tau\left[; W^{2, \infty}(\Omega)\right)^{3}$, and $\nabla \cdot v, T \in L^{q}(] 0, \tau\left[; W^{2, \infty}(\Omega)\right)$, by applying Corollary 4.6 to the space derivatives of $\rho$, it is easily checked that $\rho \in L^{\infty}(] 0, \tau\left[; W^{2, \infty}(\Omega)\right)$. As a consequence, one has that $u$ and $w$ lie in $L^{q}(] 0, \tau\left[; W^{2, \infty}\left(\Omega^{+}\right)\right)^{3}$, hence $\nabla \cdot u, \nabla \cdot w \in L^{q}(] 0, \tau\left[; W^{2, \infty}\left(\Omega^{+}\right)\right)$, and, if e. g. $\sigma(0, \cdot, m)=\nu(0, \cdot, m)=0 \quad \forall m$ on $\partial \Omega$, Theorem 3.1 can be applied.

(c) Taking into account phase transitions or encounters between droplets or ice particles would lead at least to add integral terms to the right-hand side of (7.1). Some recent studies, moreover, claims that fragmentation of big drops may play - at least in some situation - an important role (see [3] for a discussion on this topic). Taking into account also this phenomenon would lead to a modified model, and this goes beyond the scope of the present investigation too. 


\subsection{Non-differentiable solutions}

We show now by a consequentia mirabilis argument that, under suitable hypotheses, there are solutions of system (Q) that are Lipschitz continuous, but not differentiable in the space variable.

Assume $q=\infty$, that the temperature depends only on $x$ and is so low that $K_{s l}(m, T(x))=0$ and $\pi_{s}(T(x))<\pi_{l}(m, T(x)) \forall(t, x, m) \in S_{\tau}$. We also make the hypotheses that in $\Omega^{+} \sigma_{0}(x, m)=0$ and for some $\pi^{+}>0 \pi_{l}(m, T(x))-\pi_{0}(x)>\pi^{+}$, and that $v(t, x)=0$ in $Q_{\tau}$. Then there exists $\left.\left.t^{+} \in\right] 0, \tau\right]$ such that $\left.\forall t \in\right] 0, t^{+}\left[\pi(t, x)-\pi_{l}(m, T(x))<0\right.$ and hence $\sigma(t, x, m)=0$.

Assume also that, in some neighbourhood $\Omega^{0}$ of a given $x^{0}=\left(x_{1}^{0}, x_{2}^{0}, x_{3}^{0}\right)$

$$
\pi_{0}(x)=\pi_{s}(T(0, x))+x_{1}-x_{1}^{0} .
$$

Suppose now, by absurd, that $\pi$ and $\nu$ be differentiable in $x$. Integrating the equation in $\pi$, one finds, for $t \in] 0, t^{+}[$,

$$
\pi(t, x)-\pi_{s}(T(x))=E_{\nu}(t, x)\left(x_{1}-x_{1}^{0}\right)
$$

where $E_{\nu}(t, x)=e^{-\int_{0}^{t} F_{s}(\nu)\left(t^{\prime}, x\right) d t^{\prime}}$.

Assume also that $w$ is of the form $\left(0,0, w_{3}\right)$, and that $w$ and $\nabla \cdot w=\partial_{3} w_{3}$ are of class $C^{1}$.

Integrating the equation in $\nu$ along its characteristics yields then

$$
\begin{aligned}
& \nu\left(t, x_{1}, x_{2}, x_{3}(t), m(t)\right)=e^{\int_{0}^{t} L\left(t^{\prime}, x_{1}, x_{2}, x_{3}\left(t^{\prime}\right), m\left(t^{\prime}\right)\right) d t^{\prime}} \nu(0, x(0), m(0))+ \\
& +\int_{0}^{t} e^{\int_{t^{\prime}}^{t} L\left(t^{\prime \prime}, x_{1}, x_{2}, x_{3}\left(t^{\prime \prime}\right), m\left(t^{\prime \prime}\right)\right) d t^{\prime \prime}} Q_{s}(\nu, \nu)\left(t^{\prime}, x_{1}, x_{2}, x_{3}\left(t^{\prime}\right), m\left(t^{\prime}\right)\right) d t^{\prime},
\end{aligned}
$$

where, recalling also (7.2),

$$
\begin{aligned}
L & =-\partial_{3} w_{3}+\left(\frac{s_{s}}{m}-s_{s}^{\prime}\right)\left(\pi-\pi_{s}\right)-g_{s}\left[\pi-\pi_{s}\right]^{-}+J_{s}(\nu)= \\
& =-\partial_{3} w_{3}+E_{\nu}(t, x)\left[\left(\frac{s_{s}}{m}-s_{s}^{\prime}\right)\left(x_{1}-x_{1}^{0}\right)-g_{s}\left[x_{1}-x_{1}^{0}\right]^{-}\right]+J_{s}(\nu),
\end{aligned}
$$

and hence $\nu$ is not differentiable in $\left(t, x_{1}^{0}, x_{2}^{0}, x_{3}(t), m(t)\right)$ with respect to $x_{1}$.

\section{References}

[1] Ambrosio, L.: Transport equation and Cauchy problem for BV vector fields. Invent. Math. 158, 227-260 (2004)

[2] Ambrosio, L., Crippa, G., Maniglia, S.: Traces and fine properties of a BD class of vector fields and applications. Ann. Fac. Sci. XIV 4, 527-561 (2005)

[3] Barros, A.P., Prat, O.P., Testik, F.Y., Villermaux, E., Bossa, B.: Size distribution of raindrops, in Correspondence. Nat. Phys. 6, 232 (2010)

[4] Belhireche, H., Aissaoui, M.Z., Fujita Yashima, H.: Équations monodimensionnelles du mouvement de l'air avec la transition de phase de l'eau. Sciences et Technologie A (Univ. Constantine) 33, 9-17 (2011) 
[5] Belhireche, H., Aissaoui, M.Z., Fujita Yashima, H.: Solution globale de l'équation de coagulation des gouttelettes en chute. Univ. di Torino, Quaderni scientifici del Dipartimento di Matematica 5, 1-17, (2012) (to appear on Rend. Sem. Mat. Torino)

[6] Bouchut, F., James, F., Mancini, S.: Uniqueness and weak stability for multidimensional transport equations with one-sided Lipschitz coefficients. Ann. Scuola Norm. Sup. Pisa Cl. Sci. 4(5), 1-25 (2005)

[7] Cesari, L.: A boundary value problem for quasilinear hyperbolic systems in the Schauder canonic form. Ann. Scuola Norm. Sup. Pisa Cl. Sci. 1(4), 311358 (1974)

[8] Cinquini-Cibrario, M.: Sistemi di equazioni a derivate parziali in piú variabili indipendenti. Ann. di Mat. XLIV(IV), 357-418 (1957)

[9] Cinquini-Cibrario, M.: Ulteriori risultati per sistemi di equazioni quasi lineari a derivate parziali in piú variabili indipendenti. Ist. Lombardo Accad. Sci. Lett. Rend. A 103, 373-407 (1969)

[10] Conway, D.: Generalized solutions of linear differential equations with discontinuous coefficients and the uniqueness question for multidimensional quasilinear conservation laws. J. Math. Anal. Appl 18, 238-251 (1967)

[11] Di Perna, R.J., Lions, P.L.: Ordinary differential equations, transport theory and Sobolev spaces. Invent. Math. 98, 511-547 (1989)

[12] Fujita Yashima, H.: Modelación matemática del movimiento de la atmósfera con la transición de fase de agua. Revista Investigación Operacional 34(2), 93$104(2013)$

[13] Fujita Yashima, H., Campana, V., Aissaoui, M.Z.: Système d'équations d'un modèle du mouvement de l'air impliquant la transition de phase de l'eau dans l'atmosphère. Ann. Math. Afr. 2, 66-92 (2011)

[14] Jochmann, F.: A semigroup approach to $W^{1, \infty}$-solutions to a class of quasilinear hyperbolic systems. J. Math. Anal. Appl. 187, 723-742 (1994)

[15] Kato, T.: The Cauchy problem for quasi-linear symmetric hyperbolic systems. Arch. Rat. Mech. Anal. 58, 181-205 (1975)

[16] Kato, T.: Quasi-linear equations of evolution in nonreflexive Banach spaces. Lect. Notes Num. Appl. Anal. 5, 61-76 (1982)

[17] Kato, T.: Nonlinear equations of evolution in Banach spaces. Proc. Symposia Pure Math. 45(2), 9-23 (1986)(AMS)

[18] Messaadia, N., Fujita Yashima, H.: Solution stationnaire du système d'équations de la radiation et de la température dans l'air. Serdica Math. J. 39, 17-36 (2013)

[19] Selvaduray, S., Fujita Yashima, H.: Equazioni del moto dell'aria con la transizione di fase dell'acqua nei tre stati: gassoso, liquido e solido. Atti dell'Accademia delle Scienze di Torino (2011) 
[20] Tadmor, E.: Local error estimates for discontinuous solutions of nonlinear hyperbolic equations. SIAM J. Numer. Anal. 28, 891-906 (1991)

Davide Ascoli

Dipartimento di Matematica

Università di Torino

Torino

Italy

e-mail: davide.ascoli@unito.it

Steave C. Selvaduray

Dottorato di Ricerca in Matematica

Università di Torino

Torino

Italy

Received: 1 October 2012.

Revised: 25 July 2013.

Accepted: 21 August 2013. 